\title{
The triple decomposition of a fluctuating velocity field in a multiscale flow
}

\author{
P. Baj, P.J.K. Bruce, O.R.H. Buxton
}

November 2014

\begin{abstract}
A new method for the triple decomposition of a multiscale flow, which is based on the novel optimal mode decomposition (OMD) technique, is presented. OMD provides low order linear dynamics, that fits a given data set in an optimal way and is used to distinguish between a coherent (periodic) part of a flow and a stochastic fluctuation. The method needs no external phase indication since this information, separate for coherent structures associated with each length scale introduced into the flow, appears as the output. The proposed technique is compared against two traditional methods of the triple decomposition, i.e. bin averaging and proper orthogonal decomposition (POD). This is done with particle image velocimetry (PIV) data documenting the near wake of a multiscale bar array. It is shown that both traditional methods are unable to provide a reliable estimation for the coherent fluctuation while the proposed technique performs very well. The crucial result is that the coherence peaks are not observed within the spectral properties of the stochastic fluctuation derived with the proposed method however these properties remain unaltered at the residual frequencies. This proves the method's capability
\end{abstract}


of making a distinction between both types of fluctuations. The sensitivity to some prescribed parameters is checked revealing the technique's robustness. Additionally, an example of the method application for analysis of a multiscale flow is given, i.e. the phase conditioned transverse integral length is investigated in the near wake region of the multiscale object array.

\section{Introduction}

The Reynolds decomposition is commonly used in studies on turbulence to extract a stochastic fluctuation of a given quantity, which forms a basis for various subsequent analyses. This approach is justified as long as turbulence is the only source of fluctuations, however this is not necessarily the case in each situation. In a near wake region, for instance, there exists a well organized coherent large scale motion in addition to stochastic fluctuations. Treating these two as one can lead to a significant overestimation of the stochastic part of the flow.

A suitable idea for a flow decomposition in such cases was introduced by Hussain and Reynolds [1]. The authors studied an evolution of a perturbation introduced to a turbulent boundary layer at a distinctive frequency and its influence on turbulence properties. They proposed a triple decomposition having the following form:

$$
a(\mathbf{x}, t)=\bar{a}(\mathbf{x})+\tilde{a}(\mathbf{x}, \phi(t))+a^{\prime}(\mathbf{x}, t)
$$

were $a$ is an arbitrary fluctuating quantity, $\bar{a}$ is its mean value, $\tilde{a}$ is the phase dependent part (with $\phi$ denoting the phase angle) and $a^{\prime}$ corresponds to the stochastic part of $a$. Distinguishing between the two types of fluctuation is, however, nontrivial. The way Hussain and Reynolds achieved this was straight forward due to the fact that they knew the instantaneous value of the phase angle (a priori) in their experiment. Therefore they 
were able to sample only at specific phase positions (say $\phi_{0}=\phi\left(t_{0}+n \tau\right.$ ) where $\tau$ is the period) and to define $\tilde{a}$ as:

$$
\tilde{a}\left(\mathbf{x}, \phi_{0}\right)=\lim _{N \rightarrow \infty} \frac{1}{N} \sum_{n=1}^{N} a\left(\mathbf{x}, t_{0}+n \tau\right)-\bar{a}(\mathbf{x})
$$

where $N$ is the number of samples. This approach, sometimes referred to as point conditional averaging, is only possible when sampling can be aligned with the phase position, which is not the case in most situations. Because of that several other techniques for the extraction of $\tilde{a}$ have been developed since the triple decomposition was first introduced.

One of the simplest ideas for the generalization of phase averaging described above is the so called interval averaging or bin averaging introduced by Cantwell and Coles [2]. Having an external phase signal, Cantwell and Coles [2] were able to assign the phase value to each piece of data they gathered and regroup it into several subsets corresponding to different phase ranges. By performing averaging within each of those bins the authors derived an estimate for $\tilde{a}$. The procedure can be written down in the following form:

$$
\tilde{a}\left(\mathbf{x}, \phi_{0}\right)=\operatorname{mean}_{\phi(t) \in B}\{a(\mathbf{x}, t)-\bar{a}(\mathbf{x})\}
$$

where $B$ is a phase bin bounded by $\phi_{0}-\frac{\Delta}{2}$ and $\phi_{0}+\frac{\Delta}{2}$. Obviously this technique is better resolved the smaller $\Delta$ becomes but a sufficient bin width is needed to converge the statistics on the other hand. It is also important to note that large $\Delta$ causes overestimation of $a^{\prime}$ and underestimation of $\tilde{a}$. Some improvement can be achieved by following a method given in Sonnenberger et al. [3] in which $\tilde{a}$, estimated with either of the described methods, is replaced by its truncated Fourier expansion. Since $\tilde{a}$ is a periodic signal by definition no windowing is required. Sonnenberger et al. [3] showed that adopting a filtering-based method improves the convergence by a significant extent, especially when only few data points are available in each phase bin. 
A conceptually different way of decoupling $\tilde{a}$ and $a^{\prime}$ is presented in Brereton and Kodal [4]. The authors assumed the shape of the $a^{\prime}$ spectrum and then applied iterative frequency domain filtering to derive the $\tilde{a}$ signal that minimizes the cross correlation between the two fluctuation components. Importantly, no phase reference is required for this method. Coherent structures represented in (1) by $\tilde{a}$ can also be revealed by means of a proper orthogonal decomposition (POD) as presented by Perrin et al. [5]. The authors studied the near wake region behind a circular cylinder with both bin averaging and POD showing that similar structures, namely the von Kármán vortex street, can be extracted with either of these methods. However it was also noted, that phase averaging seems to overestimate the stochastic part of flow in comparison to POD. Perrin et al. [5] explains this feature with the tendency of large structures to disperse from one cycle to another, which is taken into account by POD estimation if a sufficient number of modes is used for a field reconstruction.

There are a number of other decomposition methods apart from these mentioned above (e.g. stochastic estimation by Adrian [6], wavelet analysis Grossmann and Morlet [7], empirical mode decomposition Huang et al. [8], mixed methods, etc.) but as this paper is not intended to be an exhaustive review of various triple decomposition methods, no further details will be given. One general comment ought to be made that applies to the majority of previous studies. The triple decomposition is usually considered in the case of a single scale flow (e.g. a near wake of a cylinder, single wave excitation in a turbulent boundary layer etc.). Nevertheless, bin averaging and POD are used hereafter for reference, as they are the most common triple decomposition techniques.

The focus of this paper is on multiscale flows and thereby novel in the context of the triple decomposition. Such flows can be easily recognized in nature, flow past a forest 
or an atmospheric boundary layer are good examples. There are certain phenomena coupled with some natural multiscale flows, like extremely quick forest fire propagation, that indicate their unusual properties. Recent studies on fractal generated turbulence (FGT, e.g. Hurst and Vassilicos [9] or Laizet and Vassilicos [10]) support this point as well reporting, for instance, an observation of regions of the flow in which the traditional dissipation scaling is not valid extending over considerable distances in space or a huge enhancement of turbulent mixing. One of the most noticeable features of mulitscale flows is that different coherent structures appear at different scales, each of them having their own phase and frequency, at the same instant in time. The standard triple decomposition cannot possibly account for this since it treats the coherent fluctuations as a whole. It seems though that it is crucial to make that distinction, as the coexistence and interactions of these structures are the very essence of multiscale flows. Tracking them and their evolutions can provide a significant insight into the nature of these flows, bringing us closer to an understanding of the aforementioned phenomena.

Let us propose the following formulation as an extension of the triple decomposition into multiscale flows:

$$
a(\mathbf{x}, t)=\bar{a}(\mathbf{x})+\sum_{n} \tilde{a}_{n}\left(\mathbf{x}, \phi_{n}(t)\right)+a^{\prime}(\mathbf{x}, t)
$$

This is similar to (1), except that the coherent fluctuation is split further into components belonging to different length scales introduced into the the flow. In this work we provide a suitable method of performing this decomposition (in the case of a spatiotemporal data set) based on the novel optimal mode decomposition (OMD) technique introduced by Wynn et al. [11]. OMD is the procedure of deriving low order linear dynamics that represents a given data set in an optimal way (in a sense). One can argue that it is not conceptually consistent to approximate turbulent flow with a linear model of any kind. 
This however is not our aim. The ultimate goal is to perform the triple decomposition to distinguish between the coherent (periodic) and stochastic fluctuations. Let us then use OMD purely to extract the periodic parts of the flow, namely $\tilde{a}_{n}$.

The crucial feature of our method is that no phase reference is required, moreover, the phase evolution signal, separate for each the of the considered scales, appears as the output. Traditionally, external phase indication is utilized (e.g. pressure measurement at the cylinder surface in the study of Perrin et al. [5]) to assign a value of the phase angle to each snapshot, although there are some works, where a velocity field is used to derive this quantity (see Sung and Yoo [12] for instance). In those cases however either a velocity signal at a single point is used as the reference (a lot of arbitrariness is thus introduced) or an integral quantity, e.g. circulation around the entire investigated domain, is employed. Note that this cannot be adopted in the multiscale case easily since boundaries between flow structures corresponding to different scales would be required. Our method handles the extraction of the phase information directly from the velocity field well, taking into account both phase and frequency jitter. The need for additional measurements is thereby diminished.

The proposed technique is introduced here in the context of experimental data gathered by means of particle image velocimetry (PIV), although there is no restriction for the method to experimental data. Note that all the mean fields presented hereafter need to be considered in terms of temporal averaging, i.e. no results for averaging across different experimental runs are given. 


\section{Experimental setup}

The experiments have been carried out in the open water flume facility of the Department of Aeronautics at Imperial College London. The side walls of the facility are made out of glass and therefore allow observation of the flow. The cross section of the flume has size of $600 \times 600 \mathrm{~mm}$. The background turbulence intensity is of the order of $0.9 \%$ at the center part of the flow (value normalized with local mean velocity), which was quantified in a preliminary experiment.

(a)

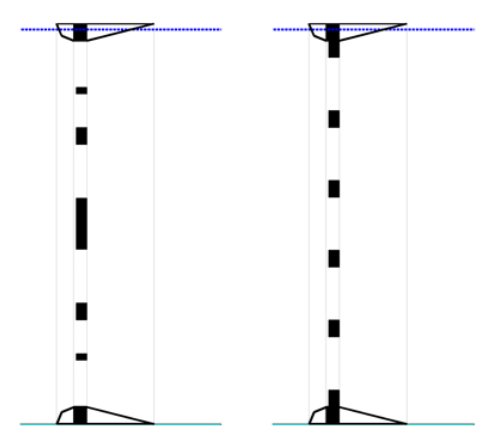

(b)

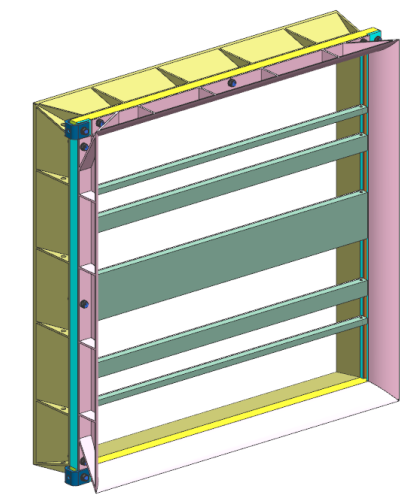

Figure 1: Grid design: a) grid I, b) grid II, c) general overview

The flow behind two different $1 \mathrm{D}$ grids (bar arrays), of which schematic views are presented in figure 1, has been examined. It can be seen that grid I represents a multiscale design while only one scale is present in the case of grid II. The bars are fixed in the outer frame (see figure 1c) having the same outer dimension as the facility's cross section. Additionally, some streamlining elements are attached to the frame in order to minimize disturbances caused by the frame, except for the $X_{0}$ station (see figure 2) where those elements would obstruct the light sheet. The blockage $\sigma$, is comparable for both grids and equals $26.7 \%$ and $27.9 \%$ respectively, based on the inner cross section of the frame (the overall blockage ratios that take into account both the grid and the mounting frame relative to the flume cross section are $38.6 \%$ and $39.6 \%$ accordingly). A ratio between 
the thickness of the thickest and the thinnest bar is equal to $t_{r}=8$ for grid I. All the measurements have been taken at the same value of free stream velocity equal to $U_{\infty}=0.2 \mathrm{~m} \mathrm{~s}^{-1}$ which results in a global Reynolds number (based on the cross section width) of $R e=120000$ and the Reynolds numbers based on bar thickness in the range of $R e_{t}=1900-15000$

(a)

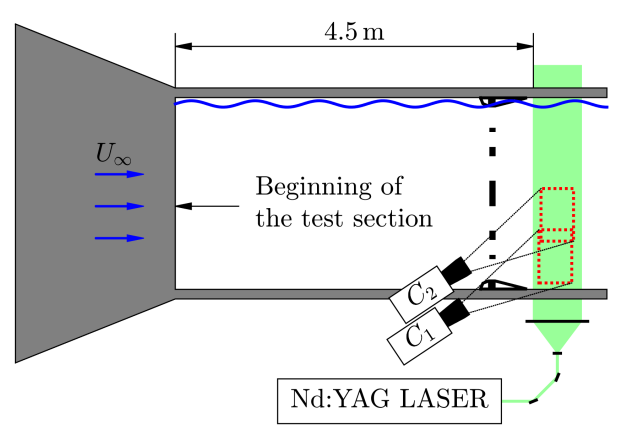

(b)

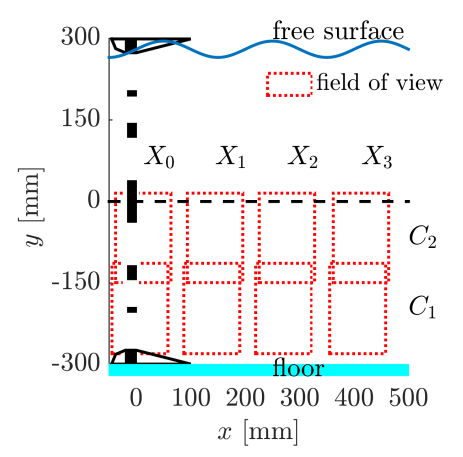

Figure 2: The experimental setup: a) general overview, b) all the measurement stations presented in the paper; they are referred to hereafter with the location labels $X_{i}$ and/or the camera field of view labels $C_{i}$

A sketch of the setup is presented in figure 2a. Cameras and optical systems have been placed at a fixed position, $4.5 \mathrm{~m}$ downstream from the beginning of the test section of the facility. The grid was mounted to a traverse that could slide in the streamwise direction. Its position was adjusted during the experiment in order to document the flow field at different downstream positions. Data corresponding to the measurements taken at the first four stations is presented in this paper (see figure $2 \mathrm{~b}$ ). The initial conditions at the extreme grid location change only slightly, i.e. a difference in background turbulence level is negligible and the boundary layer grows by $10 \%$ at that distance (as revealed by the preliminary experiment). The value of boundary layer thickness at the camera location is of the order of $75 \mathrm{~mm}$.

Two component planar PIV measurements have been carried out. At each station images have been captured simultaneously by two cameras in a side by side arrangement, 
covering an area between the floor and the centerline. Approximately $20 \mathrm{~mm}$ of overlap between the cameras' fields of view has been introduced (see figure 2b). Measurements have been taken below the centerline in order to minimize the free surface influence on the results. Both Phantom Miro M310 cameras which provide images having a resolution equal to $1280 \times 800 \mathrm{px}$, were fitted with Sigma $70 \mathrm{~mm} \mathrm{f} / 2.8$ lenses. The cameras were run at an acquisition frequency of $50 \mathrm{~Hz}$ in double frame mode (with a time delay of $\delta t=4 \mathrm{~ms}$ between frames) grabbing 1200 pairs of images at each downstream position (the acquisition time equals $48 \mathrm{~s}$ ). The flow was illuminated with an Nd:YLF laser, run at a frequency of $250 \mathrm{~Hz}$ throughout the experiment. The optical system formed a collimated laser sheet of width $190 \mathrm{~mm}$ (limited by the diameter of plano-convex lens) and thickness at the waist of order of $1 \mathrm{~mm}$.

The flow was seeded with Polyamide 12 particles having an averaged diameter of $7 \mu \mathrm{m}$ and a specific gravity equal to 1.1 . The response time $\left(\tau_{p}\right)$ was estimated by considering Stokes flow to be $3 \mu \mathrm{s}$. Making the conservative assumption that the Kolmogorov length scale in our flow is equal to $0.1 \mathrm{~mm}$ we arrive at the corresponding time scale $\tau_{\eta}=10 \mathrm{~ms}$. Therefore the Stokes number $S t=\frac{\tau_{p}}{\tau_{\eta}}=3 \cdot 10^{-4} \ll 1$ ensures that the particles behave as tracers and track the smallest scale motions of the flow.

Cross correlation calculations were performed with the commercial software of LaVision GmbH: Davis 8.2. An interrogation window of $24 \times 24$ px with $75 \%$ overlap has been applied with a multipass approach and a median filter to replace spurious vectors (less then $3 \%$ ). The resulting resolution is equal to $3.2 \mathrm{~mm}$ with a vector spacing of $0.8 \mathrm{~mm}$. The noise level of the rms values of velocity has been evaluated according to the procedure provided in Romano et al. [13] to be equal to $0.5 \%$. 


\section{Triple decomposition: the traditional approach}

Let us focus on the mean flow topology behind the multiscale grid first. The averaged streamwise velocity and turbulence intensity are presented in figure 3. The wakes of different bars are clearly distinguishable close behind the grid and become merged at some point further downstream. The initial intersection point can be intuitively recognized during visual inspection of the figure as:

- being located within the second downstream station in the case of the smallest and the medium bar

- being located within the fourth downstream station in the case of the medium and the biggest bar

(a)

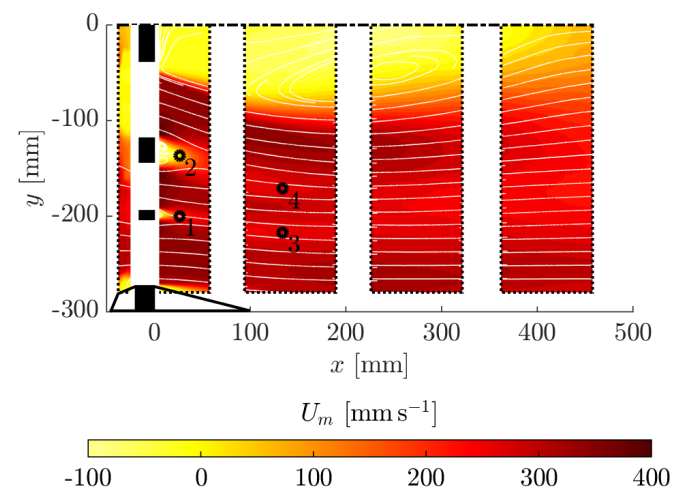

(b)

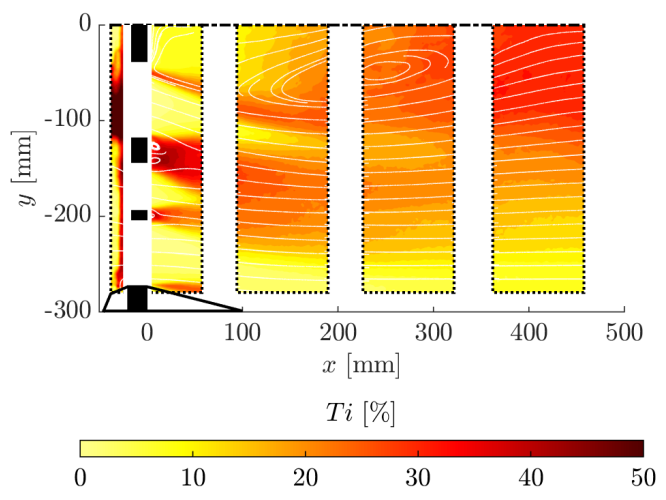

Figure 3: The results for grid I: a) mean streamwise velocity (numbered circles indicates PSD evaluation positions), b) turbulence intensity

The near wake can be naturally split with the intersection point into two regimes, each of them interesting. This distinction is important from the method validation point of view since extracting spatially separated coherent structures is a qualitatively different task to extracting merged patterns. It is worth evaluating the method efficiency in both cases. It seems, that the interaction of the smallest and the medium bar is better suited 
for further analysis because both regions are gathered within our data, therefore we stick to this example hereafter.

(a)

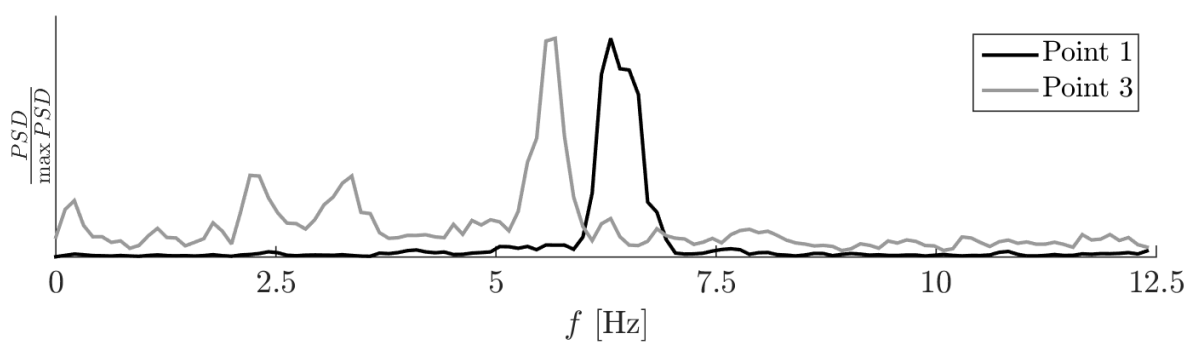

(b)

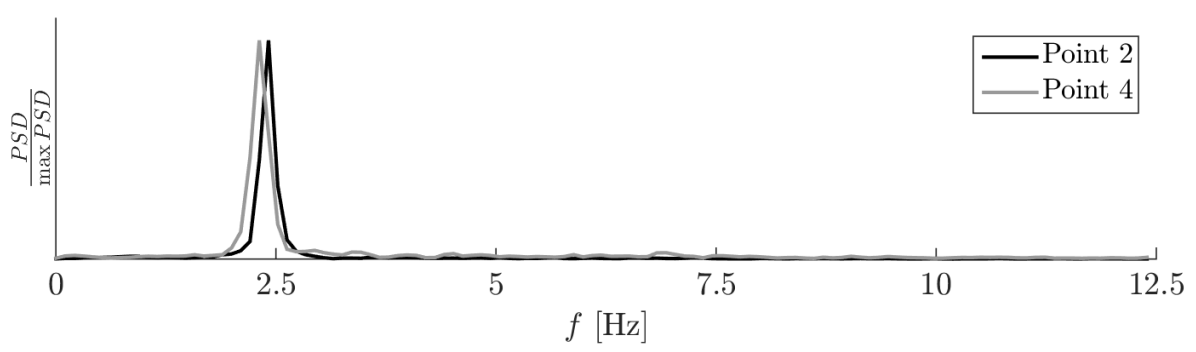

Figure 4: Normalized power spectral density evaluated for the transverse velocity fluctuation behind: a) the small bar, b) the medium bar (see figure 3a for exact location)

Coherent structures in a flow past a cylinder are well recognized in the literature. The regular vortex street, shed at a narrow frequency band, forms behind the body and breaks down into turbulence further downstream (if the Reynolds number is sufficient). In order to avoid major concerns in the following sections, let us highlight the discrepancy between such a classical situation and our case. The power spectral density (PSD) has been evaluated for the transverse velocity component (see figure 4) at four different points whose positions are denoted in figure 3 a by numbered circles. The points are located at the wakes' centerlines within the first two measurement stations. The resulting shedding parameters are summarised in table 1. The Strouhal number based on inlet velocity and bar thickness is significantly higher (see table 1) compared to the usually reported values (Okajima [14] for instance), however this can be linked to the blockage effect reported by West and Apelt [15]. A simple blockage ratio correction applied to the velocity value 
Table 1: Parameters of the shedding of the medium and the small bar (see figure 1a), $A R$ stands for aspect ratio of a bar cross section and $\delta$ for bar thickness

\begin{tabular}{c|cc|cc} 
& \multicolumn{2}{|c|}{ small bar } & \multicolumn{2}{|c}{ medium bar } \\
& $X_{0}$ & $X_{1}$ & $X_{0}$ & $X_{1}$ \\
\hline$A R$ & 0.63 & \multicolumn{2}{|c}{1.68} \\
$R e_{t}$ & 1900 & \multicolumn{2}{|c}{5100} \\
$f_{s h}[\mathrm{~Hz}]$ & 5.70 & 6.54 & 2.40 & 2.32 \\
$S t=\frac{f_{s h} \cdot \delta}{U_{\infty}}$ & 0.27 & 0.31 & 0.30 & 0.29 \\
$\frac{S t}{(1-\sigma)}$ & 0.17 & 0.19 & 0.19 & 0.19
\end{tabular}

produces Strouhal numbers much more consistent with accepted values $(\simeq 0.15)$.

The most striking fact however is that there is a considerable drop in the shedding frequency of the smaller bar between the sampling points (the change is of the order of $15 \%)$. This feature is noticeable in all the spectral data presented hereafter. It is a result of the rear streamlining elements being detached during measurements at the $X_{0}$ station which can be seen as an increase in the blockage ratio due to pronounced areas of detached flow behind the frame bars. The higher shedding frequency observed at this position is therefore consistent with West and Apelt [15] results.

\subsection{Bin averaging method}

In our case there are two wakes (behind the small and the medium bar) enclosing distinctive coherent structures and, as indicated by (4), we are aiming to extract them separately. Let us apply the bin averaging procedure, similar to the one used in Cantwell and Coles [2] or Perrin et al. [5] (although single scale cases are considered there), for this purpose first. This method requires a reference phase signal or, being more precise, an individual 
(a)

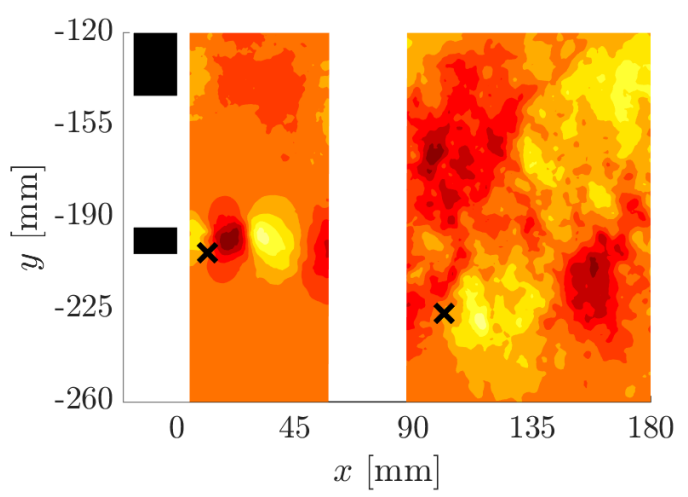

(b)

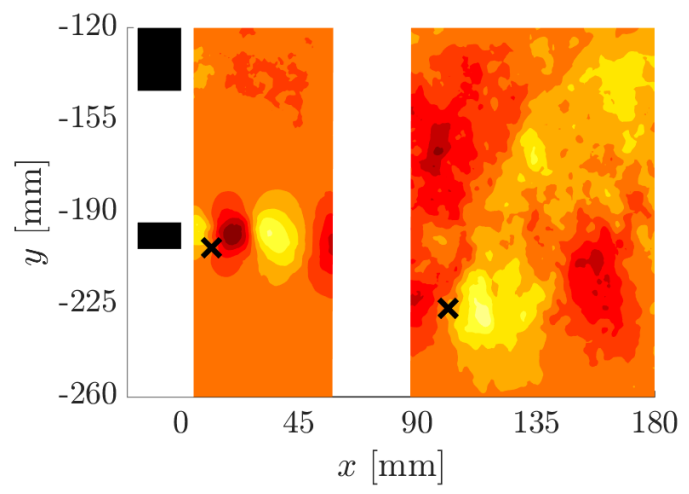

Figure 5: The normalized bin averaging results based of the phase signal evaluated at the points marked with x signs: a) the simple method, b) the truncated Fourier series method

reference for each wake. In the previously mentioned studies pressure measurements on a cylinder surface are taken as such but in our case the PIV data is the only available. Therefore the velocity signal at an arbitrary point within a certain wake is utilized to produce the reference signal. The Hilbert transform (a convolution of the signal with a function $h(t)=\frac{1}{\pi t}$, see Perrin et al. [5] for reference) has been used to recover the instantaneous phase value from the transverse velocity fluctuation.

The example results of bin averaging are shown in figure $5 \mathrm{a}$ (wake behind the smallest bar for an arbitrary phase value). The reference points are marked with the $\mathrm{x}$ symbol (one for each downstream station). The bin size has been set to $0.2 \mathrm{rad}$, which results in approximately 35 images per bin over which the statistics can be calculated hence the mean values are rather poorly converged. In order to improve the results a truncated Fourier expansion has been used (see Sonnenberger et al. [3]) with the cutoff frequency set to double that of the shedding frequency. This output is depicted in figure 5b.

It can clearly be seen in figure 5 that the part of the transverse velocity fluctuation conditioned on the smallest bar shedding phase reaches significant values within the wake of the medium bar. This effect is more pronounced in the case of figure 5a. In order 


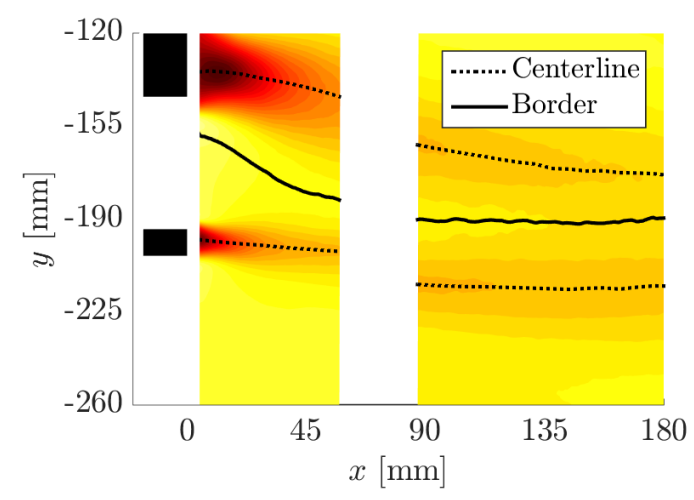

Figure 6: An interface between wakes' areas and wakes' centerlines, local extrema of the mean streamline velocity field, utilized hereafter to quantify results

to quantify this effect fields of view were split with a line following local maxima of the mean velocity field (localized between the wakes, see figure 6) into two areas associated with each of the wakes. The coherent fluctuation's energy corresponding to either bar can be thus decomposed at each downstream station, based on the field of view division, into a share that falls into the associated region, denoted by $E_{s}$, and the remaining part (this is summarised in the table 2). Additionally, let us define a length scale $z$ that is a second moment of the energy around a wake's centerline ( $e$ stands for the local coherent fluctuation energy and $y_{c}$ for the centerline coordinate). This is a measure of variance of the coherent fluctuation's energy distribution around the centerline averaged across the field of view.

$$
z=\sqrt{\frac{\sum_{x} \sum_{y}\left[y-y_{c}(x)\right]^{2} e(x, y)}{\sum_{x} \sum_{y} e(x, y)}}
$$

The initial observation is supported by the presented metrics. More than half of the small bar shedding energy at the $X_{1}$ station is contained in the area associated with the other wake. On the other hand, a significant amount of the medium bar shedding energy is missing at the $X_{0}$ station. Another problem is that $z$ evaluated for the medium wake decreases with the downstream position which is nonphysical, as structures are observed to grow in size with a downstream position (e.g. Cantwell and Coles [2], Perrin et al. [5]). 
Table 2: Parameters of an energy distribution of the coherent fluctuation of transverse velocity extracted with bin averaging ( $\delta$ stands for bar thickness)

\begin{tabular}{c|cc|cc} 
& \multicolumn{2}{|c|}{$E_{s}$} & \multicolumn{2}{|c}{$\frac{z}{\delta}$} \\
& $X_{0}$ & $X_{1}$ & $X_{0}$ & $X_{1}$ \\
\hline small bar & $91.8 \%$ & $46.3 \%$ & 2.02 & 4.71 \\
medium bar & $74.6 \%$ & $90.0 \%$ & 1.43 & 0.78
\end{tabular}

It is thus justifiable to state that the simple bin averaging procedure cannot be applied to decouple coherent structures belonging to different sized bars.

\subsection{POD method}

Let us move on to the triple decomposition based on proper orthogonal decomposition (POD). The general formula of this decomposition is given below:

$$
a(\mathbf{x}, t)=\sum_{n=1}^{N} c_{n}(t) \cdot \Phi_{n}^{P O D}(\mathbf{x})
$$

were $\Phi_{n}^{P O D}$ is the $n$-th POD mode and $c_{n}$ is its corresponding time dependent coefficient. Extraction of the POD modes is done by considering an eigenvalue problem for the velocity fluctuation correlation matrix. As a result of such an approach the POD modes are ranked by their energy content. POD is an optimal decomposition in a sense that it minimizes the number of modes required to reconstruct a set of data for a given error level. For more details on POD see the extensive review of Berkooz et al. [16].

The triple decomposition (1) and the POD formula (6) are quite different, however it was reported in the past (by Perrin et al. [5] for instance) that in the case of a flow behind a cylinder at a sufficiently high Reynolds number the first few POD modes can be directly linked to the von Kármán vortex street. Usually, in a single cylinder study, the first two modes carry the essential information (e.g. $60 \%$ of total fluctuation's energy 
in the case of Perrin et al. [5]). Since a vortex street is a phase dependent structure we would like to extract, it seems that POD analysis may fit our needs.

The POD approach has proven to be efficient in separating the shedding pattern of the medium bar (calculations were done separately for each station), as the first two POD modes clearly correspond to the vortex street of the medium bar. Contrary to what has been observed in the case of bin averaging, the wake area of the smallest bar is not occupied by structures belonging to the shedding of the medium bar which can be confirmed by the high energy contained in the medium wake area (see table 3). On the other hand, POD has not been able to separate the shedding of the small bar. There is no indication for which modes contain the associated pattern. Our intuition was that the third and the fourth modes would carry this information since the smaller wake is less energetic than the medium one but still contains a significant proportion of the total energy. This has turned out to be the case at the first station, where the wakes are clearly separated (see figure 7). Both the shape of those modes and associated PSD clearly point to the shedding pattern. In the case of the $X_{1}$ station however the aforementioned intuition has failed. None of modes $3-6$, presented in figure 7 , can be directly linked to the shedding and further modes do not bring any additional insight. Modes 4 and 6 resemble a shedding pattern to a large extent, however the associated spectra contain multiple peaks which should not occur in the case of properly extracted shedding fluctuations. If one were to treat them as such the corresponding energy share falling into the proper wake area would be low (see table 3).

The given example pushes us towards the belief that POD is not well suited for triple decomposition in multiscale flow either. It is a well known fact (see Graham and Kevrekidis [17]), that POD deemphasizes flow features that carry relatively little energy 

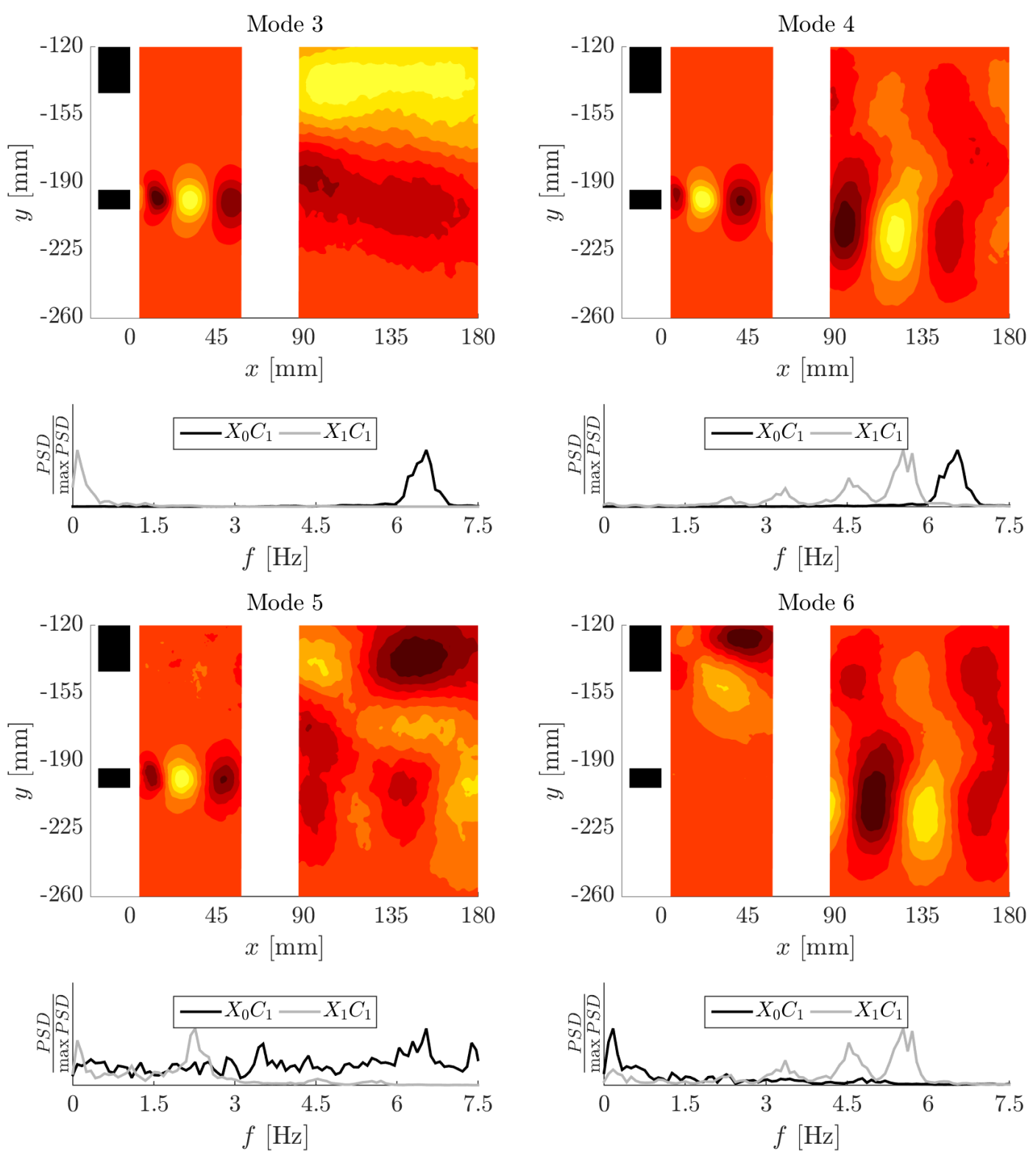

Figure 7: The 3rd to the 6th POD modes of the transverse velocity component with the spectra of corresponding coefficients

Table 3: Parameters of an energy distribution of the coherent fluctuation of transverse velocity extracted with POD ( $\delta$ stands for bar thickness, parameters of the small bar shedding at $X_{1}$ stations evaluated with modes 4 and 6 )

\begin{tabular}{|c|c|c|c|c|}
\hline & \multicolumn{2}{|c|}{$E_{s}$} & \multicolumn{2}{|c|}{$\frac{z}{\delta}$} \\
\hline & $X_{0}$ & $X_{1}$ & $X_{0}$ & $X_{1}$ \\
\hline small bar & $99.5 \%$ & $64.5 \%$ & 0.77 & 3.58 \\
\hline medium bar & $98.3 \%$ & $90.9 \%$ & 0.47 & 0.76 \\
\hline
\end{tabular}


regardless of their dynamical importance. It seems, therefore, that the failure in our case can be linked to a significant difference in energy content between the two wakes. Treating the results for the first station as the reference for such a comparison, the medium wake contains $30.8 \%$ of total fluctuation energy while the small one only $6.5 \%$ (additional information, based on the developed method, is given in section 6). On the other hand, there is no strict premise for treating the energy content as a proper filter for making a distinction between coherent structures associated with different scales. The actual reason for the POD failure is, however, of the secondary matter here because a technique well suited for the task is presented below.

\section{Triple decomposition: the DMD/OMD approach}

A new decomposition technique called dynamic mode decomposition (DMD) was proposed recently by Schmid [18]. Similar to POD, DMD is a snapshot based method. The author aimed to develop an approach that allows better insight into the dynamics of a process than POD does. The fundamental idea behind DMD is that there is a linear dependency, approximately constant throughout the sampling period, between successive snapshots. This can be written as:

$$
\begin{gathered}
\mathbf{v}_{n+1}=\mathbf{A} \mathbf{v}_{n} \\
\mathbf{A} \in \mathbb{R}^{p \times p}
\end{gathered}
$$

where $\mathbf{v}_{n}$ stands for an arbitrary snapshot (having length denoted by $p$ ) while $\mathbf{A}$ represents the underlying linear system. Evidently, $\mathbf{v}_{n}$ is a $1 \mathrm{D}$ vector, nevertheless it represents a $2 \mathrm{D}$ snapshot and the straight forward transformation between both forms exists. The DMD procedure estimates the dynamic properties of $\mathbf{A}$ and its successful adaptation relies on having a sufficiently large ensemble of snapshots. It is worth noting that in a usual 
application (say to PIV data) the matrix $\mathbf{A}$ is of a considerable size hence its analysis is nontrivial. The standard least square approach results in the following expression for $\mathbf{A}$ ( $N$ is the number of available snapshots):

$$
\min _{\mathbf{A}}\left\|\left[\mathbf{v}_{2}, \ldots, \mathbf{v}_{N}\right]-\mathbf{A} \cdot\left[\mathbf{v}_{1}, \ldots, \mathbf{v}_{N-1}\right]\right\|^{2}
$$

(8) is an ill-posed problem since the number of unknowns exceeds the number of the matrix's elements for which the norm is to be minimized. Assuming that an arbitrary snapshot is a linear combination of the remaining ones Schmid [18] was able to overcome this difficulty and restated the problem in the following way:

$$
\begin{gathered}
\min _{\mathbf{S}}\left\|\left[\mathbf{v}_{2}, \ldots, \mathbf{v}_{N}\right]-\left[\mathbf{v}_{1}, \ldots, \mathbf{v}_{N-1}\right] \cdot \mathbf{S}\right\|^{2} \\
\mathbf{S} \in \mathbb{R}^{N-1 \times N-1}
\end{gathered}
$$

where $\mathbf{S}$ is the companion matrix of significantly lower rank. Nevertheless, the eigenvalues of $\mathbf{S}$ approximate those of $\mathbf{A}$.

A generalized form of DMD, called optimal mode decomposition (OMD), was introduced by Wynn et al. [11]. The OMD method approaches (8) in a more straight forward way, replacing $\mathbf{A}$ with its low rank approximation $\mathbf{L} \mathbf{M L}{ }^{T}$. The problem then takes the form:

$$
\begin{gathered}
\min _{\mathbf{L}, \mathbf{M}}\left\|\left[\mathbf{v}_{2}, \ldots, \mathbf{v}_{N}\right]-\mathbf{L} \mathbf{M} \mathbf{L}^{T} \cdot\left[\mathbf{v}_{1}, \ldots, \mathbf{v}_{N-1}\right]\right\|^{2} \\
\mathbf{L}^{T} \mathbf{L}=\mathbf{I} \\
\mathbf{L} \in \mathbb{R}^{p \times r} \quad \mathbf{M} \in \mathbb{R}^{r \times r}
\end{gathered}
$$

where both $\mathbf{L}$ and $\mathbf{M}$ are treated as independent variables in an optimization process with an arbitrary rank constraint $r\left(r=\operatorname{rank}(\mathbf{M})=\operatorname{rank}\left(\mathbf{L} \mathbf{M} \mathbf{L}^{T}\right)\right)$. L can be seen as a low dimensional subspace of the flow field while $\mathbf{M}$ gathers the corresponding dynamics. Wynn et al. [11] proved that OMD turns into DMD if one sets $\mathbf{L}$ to the POD modes 
explicitly which is the major limitation of DMD, since POD modes do not necessarily form the optimal basis for dynamic information extraction.

Since DMD is the restricted version of OMD, the following remarks apply equally to both methods. The OMD eigenvalues are defined as

$$
\lambda_{n}^{O M D}=\frac{\log \lambda_{n}(\mathbf{M})}{\Delta t}
$$

where $\lambda_{n}(\mathbf{M})$ are eigenvalues of the matrix $\mathbf{M}$ and $\Delta t$ is a reversal of the sampling frequency. The OMD modes associated with $\lambda_{n}^{O M D}$ values follow the formula:

$$
\Phi_{n}^{O M D}(\mathbf{x})=\mathbf{L} \mathbf{z}_{\mathbf{n}}
$$

where $\mathbf{z}_{\mathbf{n}}$ is an eigenvector corresponding to the $\lambda_{n}(\mathbf{M})$. A flow field can be reconstructed (up to the error due to the linear modeling) in the following manner:

$$
a(\mathbf{x}, t) \simeq \sum_{n=1}^{r} c_{n} \Phi_{n}^{O M D}(\mathbf{x}) e^{\lambda_{n}^{O M D} t}
$$

where $c_{n}$ are coefficients associated with the OMD modes. The coefficients appear in (13), since $\Phi_{n}^{O M D}$ are given in a normalized form $\left(\left\|\Phi_{n}^{O M D}\right\|^{2}=1\right) .\left|c_{n}\right|$ is referred to as the DMD spectrum hereafter. The important note is that both DMD and OMD methods produce pairs of conjugate modes since we deal with real data. Presenting OMD/DMD results hereafter we make no distinction between them (i.e. when we refer to a single mode we mean a conjugated pair of modes in fact).

\subsection{Application of the DMD}

Let us start with the classical DMD, as described by Schmid [18]. The technique has been applied to the data gathered at stations $X_{0} C_{1}$ and $X_{1} C_{1}$ (see figure $2 \mathrm{~b}$ ). DMD spectra for both stations are presented in figure 8 together with transverse velocity spectra enveloped 
across the entire field of view denoted by $F_{v}$ (referred to as enveloped spectra hereafter). The latter is defined as follows:

$$
F_{u}(f)=\max _{\mathbf{x}} \hat{u}(\mathbf{x}, f)
$$

where $\hat{u}$ is PSD of an arbitrary signal $u=u(\mathbf{x}, t)$ ( $f$ stands here for frequency). Both spectra appear to be similar, although the shedding peak of the small bar is less pronounced in the case of the DMD spectrum. Frequencies corresponding to the local maxima of the DMD spectra within the previously recognised (see figure 4) shedding bands are highlighted and the associated DMD modes are depicted in figure 9. A great deal of improvement is achieved in the vortex street pattern recognition compared to previously tested methods; concerning the small bar modes in particular, the coherent fluctuations' energy share in the associated area reaches $95.7 \%$ at the $X_{1}$ station (see table 4 ). On the other hand results seems to be better at the $X_{0}$ station in the case of POD (decreased energy content and increased $z$ scale), which is due to a high level of noise that can be observed in the medium wake area.

(a)
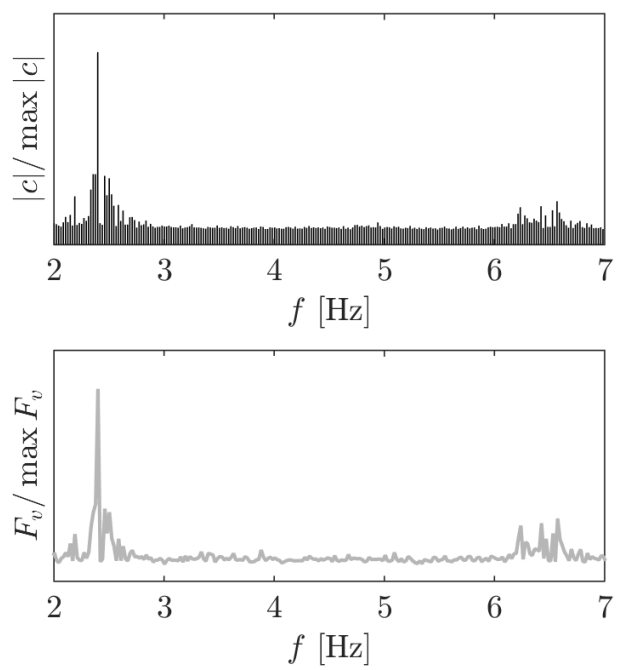

(b)
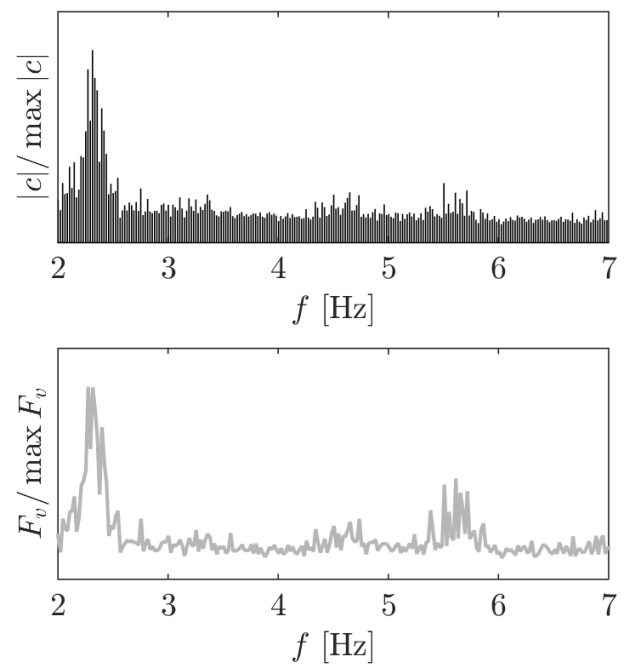

Figure 8: Normalized DMD spectra (above) and corresponding enveloped transverse velocity spectra for: a) station $X_{0} C_{1}$, b) station $X_{1} C_{1}$ 
(a)

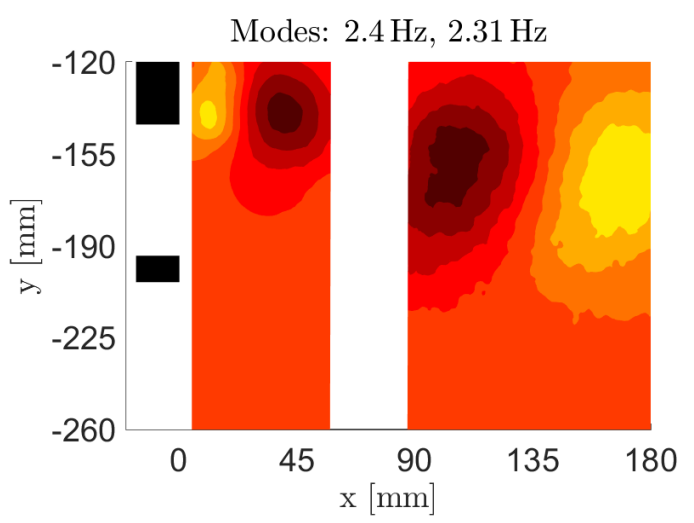

(b)

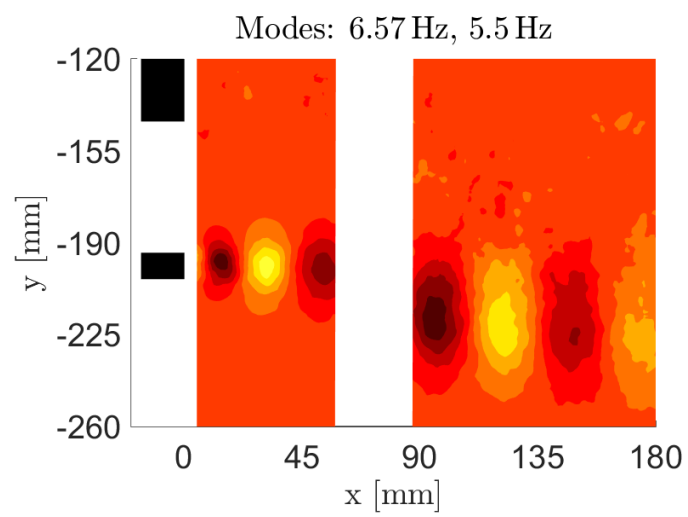

Figure 9: DMD modes corresponding to the local maxima of the DMD spectra (highlighted in figure 8) localised in: a) the shedding band of the medium bar, b) the shedding band of the small bar

Table 4: Parameters of an energy distribution of the coherent fluctuation of transverse velocity extracted with DMD ( $\delta$ stands for bar thickness)

\begin{tabular}{c|cc|cc} 
& \multicolumn{2}{|c|}{$E_{s}$} & \multicolumn{2}{|c}{$\frac{z}{\delta}$} \\
& $X_{0}$ & $X_{1}$ & $X_{0}$ & $X_{1}$ \\
\hline small bar & $97.4 \%$ & $95.7 \%$ & 1.28 & 1.82 \\
medium bar & $98.4 \%$ & $92.3 \%$ & 0.46 & 0.78
\end{tabular}

Although the aforementioned results are promising, no direct link to $\tilde{a}_{n}$ has been shown so far. It is clear from figures 4 and 8 that shedding peaks are of considerable width rather than limited to distinctive frequencies. Therefore the simplest idea for extracting the coherent parts is to apply (13) and perform the summation over only those modes with corresponding frequencies that fall into the shedding bands. There are two major drawbacks to this approach:

- The spectra of stochastic fluctuations would be almost entirely suppressed within those frequency bands (up to the decomposition error)

- Only a few of the considered modes can be directly linked to the shedding 
(a)

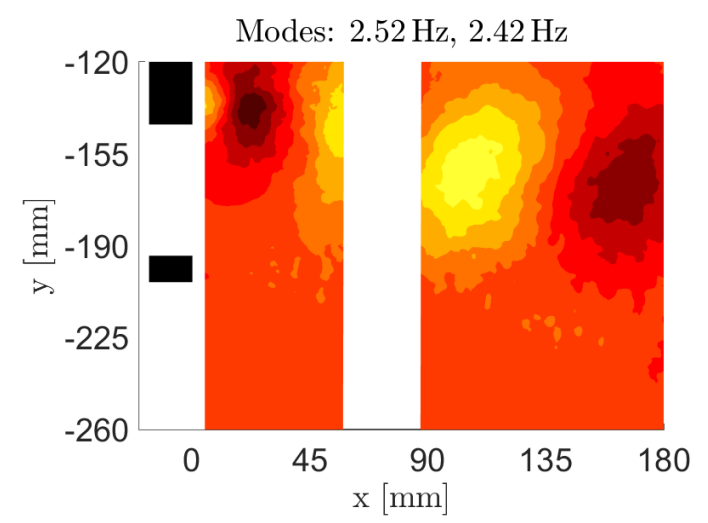

(b)

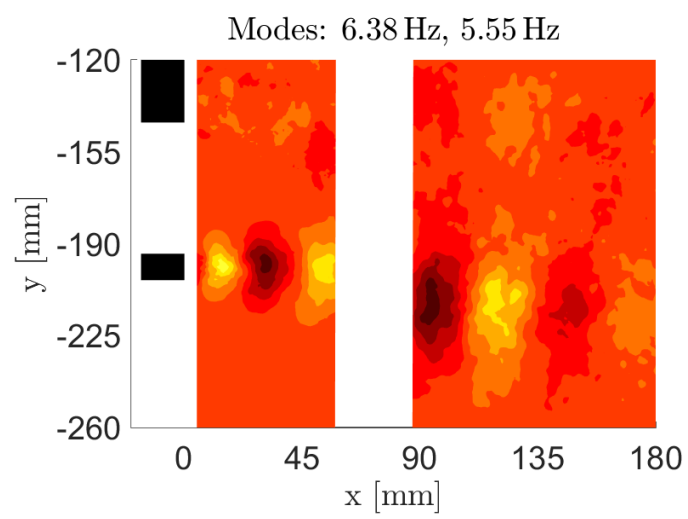

Figure 10: DMD modes corresponding to frequencies slightly off the local maxima of the DMD spectra (highlighted in figure 8) localised in: a) the shedding band of the medium bar, b) the shedding band of the small bar

The first issue is particulary hard to address. Some kind of distinction between the coherent and stochastic fluctuations share within each mode shall be introduced but this is simply a step back to the initial problem. The second matter is also worrisome. Modes presented in figure 9, despite some secondary features, can be clearly associated with shedding. This however can't be said about the modes shown in figure 10 for instance although their frequencies fall into the shedding bands as well (but are towards the sides). It is clear that some strong non shedding events infect these modes, especially those belonging to the small bar (the energy share in the associated area is limited to $81.3 \%$ at the $X_{1}$ station and the $z$ scale increases almost twice). This indicates the fact that an additional selection is required in order to filter only "true" shedding modes. Such selection would introduce a great deal of arbitrariness into the method, which is highly undesirable.

\subsection{Application of the OMD}

The OMD method, by its very definition, requires the rank constraint to be set to a certain value explicitly. In the DMD approach this is done implicitly by posing the problem in the 
form (9), which introduces the constraint $r=N-1$. Since the OMD procedure is much more computationally expensive and both size and number of snapshots are large in our case, the subspace rank for the OMD approach has to be significantly lower. Moreover, it is assumed that choosing a comparable $r$ value will result in a similar output. Given that, it was decided to set the rank constraint at a level of $20 \leq r \leq 30$.

(a)

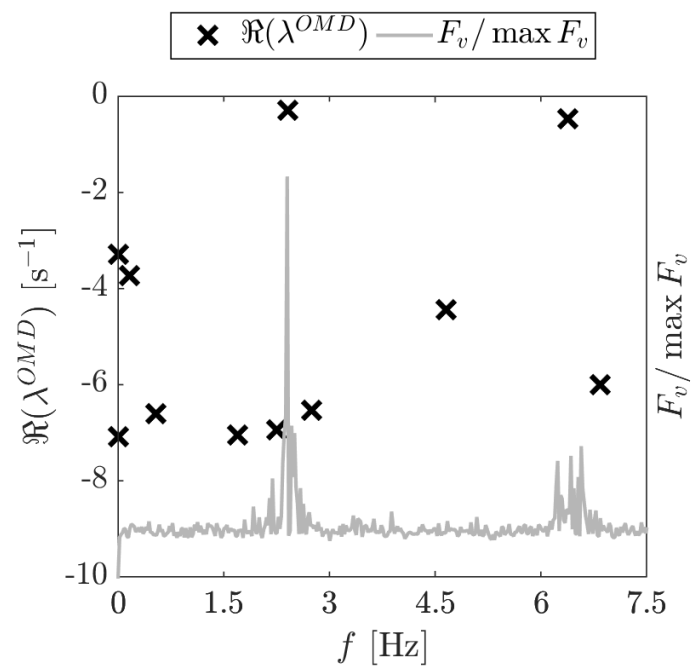

(b)

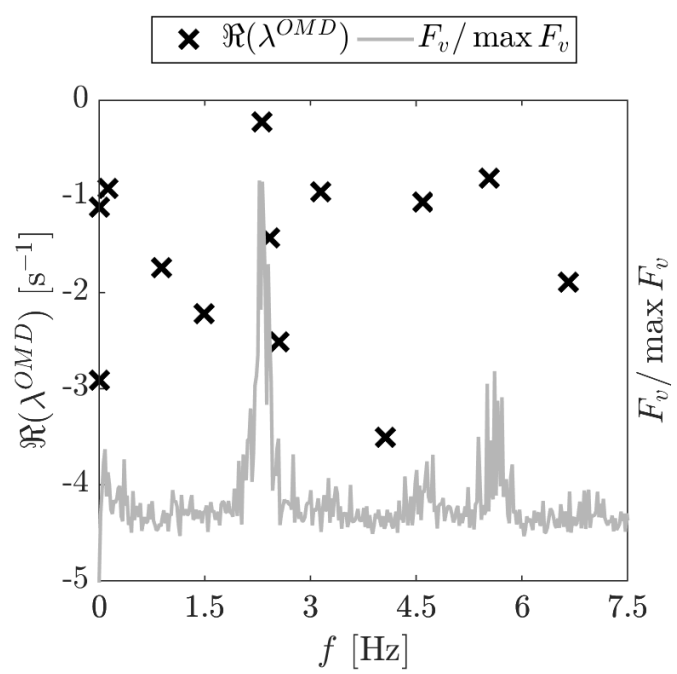

Figure 11: Real part of OMD eigenvalues with the enveloped transverse velocity spectra in the background for: a) station $X_{0} C_{1}$, b) station $X_{1} C_{1}$

(a)

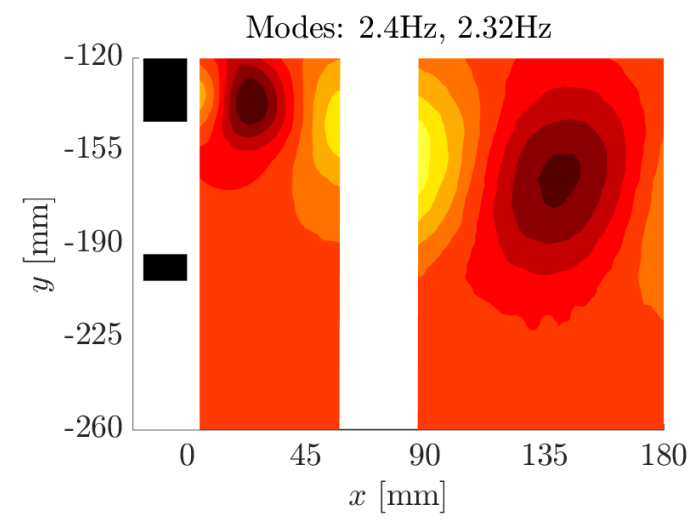

(b)

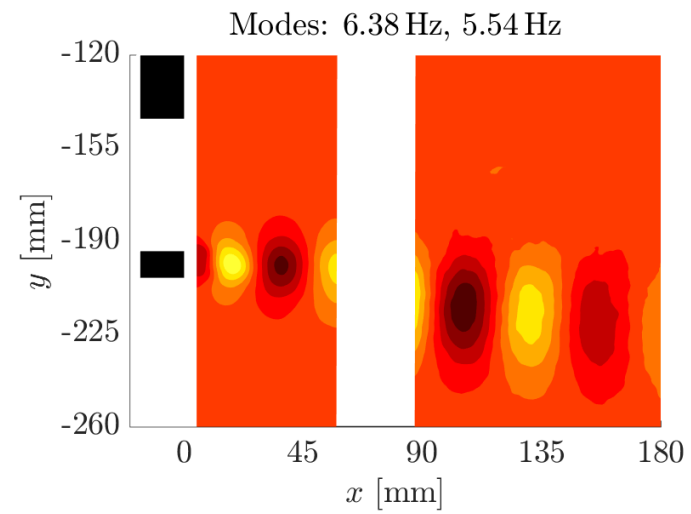

Figure 12: OMD modes corresponding to the local maxima of $\Re\left\{\lambda^{O M D}\right\}$ localised in: a) the shedding band of the medium bar, b) the shedding band of the small bar

The case investigated with DMD in the previous section is analysed here with OMD. OMD eigenvalues, derived with $r=24$ (the method's sensitivity to this parameter is 
investigated in section 5.1), are given in figure 11 (presented in frequency - damping space), with the transverse velocity spectra enveloped across the field of view shown in the background. There are distinctive OMD modes appearing at the shedding frequencies in which the associated damping (equal to $\left|\Re\left\{\lambda_{n}^{O M D}\right\}\right|$ ) is recognizably low. Shapes of those modes are presented in figure 12. Again, it is clear from visual inspection that they correspond to shedding patterns. Compared to figure 9, the considered modes are less noisy which can be confirmed by the data gathered in table 5 . It can be seen that OMD recovers the largest amount of the small bar shedding energy within the corresponding area compared to other methods and that it makes the small bar shedding the most confined (the smallest $z$ values).

Table 5: Parameters of an energy distribution of the coherent fluctuation of transverse velocity extracted with OMD ( $\delta$ stands for bar thickness)

\begin{tabular}{|c|c|c|c|c|}
\hline & \multicolumn{2}{|c|}{$E_{s}$} & \multicolumn{2}{|c|}{$\frac{z}{\delta}$} \\
\hline & $X_{0}$ & $X_{1}$ & $X_{0}$ & $X_{1}$ \\
\hline small bar & $99.6 \%$ & $97.2 \%$ & 0.76 & 1.62 \\
\hline medium bar & $98.2 \%$ & $90.6 \%$ & 0.47 & 0.75 \\
\hline
\end{tabular}

As in the case of the DMD based investigation, there are other OMD modes occurring at the frequencies falling into shedding bands. Therefore a doubt rises, that parts of coherent structures are carried by those modes as well. In order to address this issue the neighbours of the modes given in figure 12 are examined here. The corresponding shapes are given in figure 13. Although the frequencies of some of the presented modes are located well within the shedding bands, none of the shapes recall primary vortex street features. This shows that, unlike in the DMD case, only one mode can be directly linked to each shedding. This is a significant improvement compared to the DMD technique, since no 
additional mode selection is necessary and the correct mode can be easily selected based on $\Re\left\{\lambda^{O M D}\right\}$ local maxima.

(a)

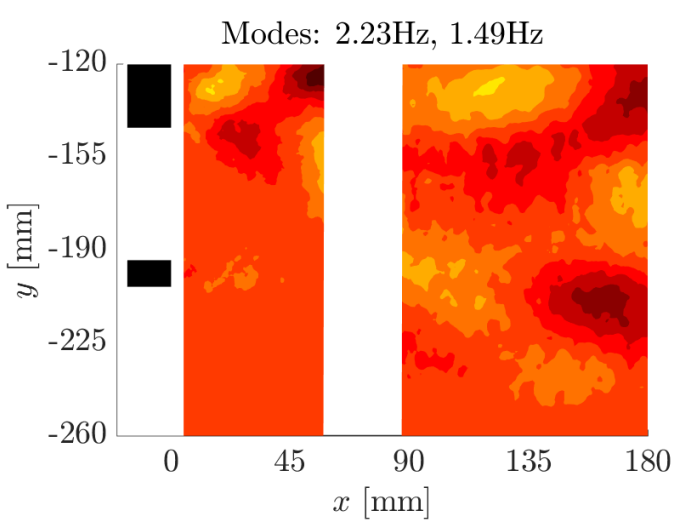

(c)

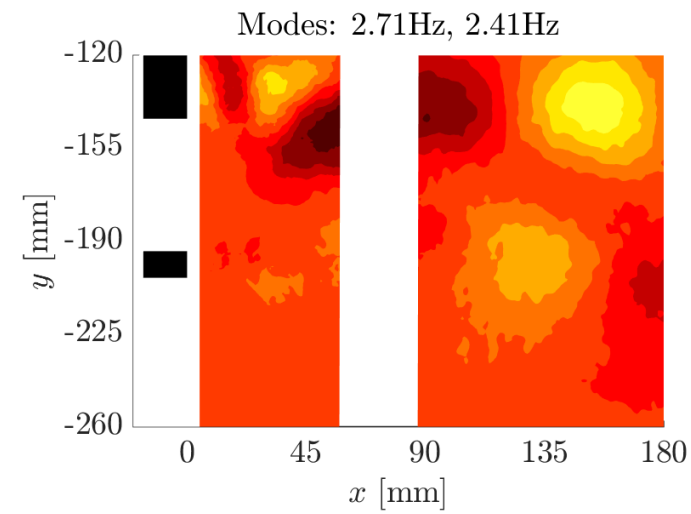

(b)

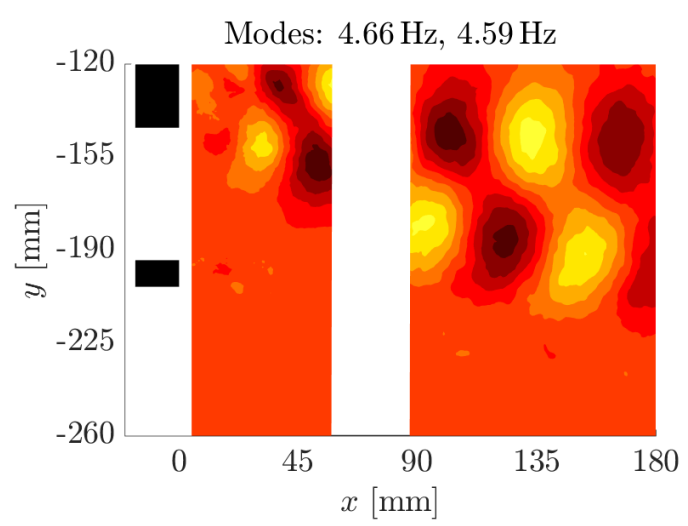

(d)

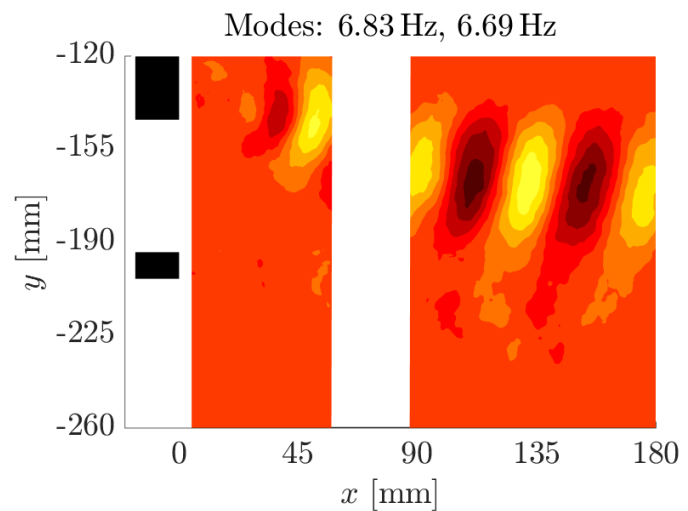

Figure 13: Neighborhood of OMD modes corresponding to local maxima of $\Re \lambda^{O M D}$ : a) previous to $12 \mathrm{a}, \mathrm{b}$ ) previous to $12 \mathrm{~b}, \mathrm{c})$ subsequent to $12 \mathrm{a}, \mathrm{d}$ ) subsequent to $12 \mathrm{~b}$

OMD appears to be the most capable method of identifying coherent structures occurring simultaneously at different frequencies in a multiscale flow. Nevertheless, extracting the phase dependant part of the flow is still nontrivial because it turns out that the straight forward way, namely utilizing (13), leads to a solution that vanishes in time. The damping associated with the $2.4 \mathrm{~Hz}$ mode, for instance, is of the order of $0.29 \mathrm{~s}^{-1}$. Since the sampling time is equal to $48 \mathrm{~s}$, the final gain corresponding to this particular mode is of order of $10^{-6}$ (or even less in other cases). This result indicates a conclusion that the coherent structures of the near wake region are strongly damped which is in clear contra- 
diction to the physical knowledge (a vortex street is known to be periodic throughout the flow time if all the parameters are fixed). Therefore its obvious extraction method can't be directly applied.

\section{Triple decomposition: the proposed approach}

In order to overcome the difficulty with utilizing OMD for the triple decomposition a new approach has been established. It is proposed to perform a projection of the original snapshots onto OMD modes. This can be written as:

$$
\begin{gathered}
\min _{\mathbf{d}_{1}, \ldots, \mathbf{d}_{r}}\left\|\left[\mathbf{v}_{1}, \ldots, \mathbf{v}_{N}\right]-\left[\Phi_{1}^{O M D}, \ldots, \Phi_{r}^{O M D}\right] \cdot\left[\mathbf{d}_{1}, \ldots, \mathbf{d}_{r}\right]^{T}\right\|^{2} \\
\mathbf{d}_{n} \in \mathbb{C}^{N \times 1}
\end{gathered}
$$

where $\mathbf{d}_{n}$ is a vector of coefficients corresponding to the $\Phi_{n}^{O M D}$. In order to reconstruct the phase dependent part of flow one can write:

$$
\tilde{a}(\mathbf{x}, \phi(t))=\sum_{\substack{\text { shedding } \\ \text { modes }}} d_{n}(t) \cdot \Phi_{n}^{O M D}(\mathbf{x})
$$

This simple idea recalls the POD approach, except that instead of the standard POD modes, the OMD modes are used to calculate the corresponding coefficients and to reconstruct the field later on.

In order to examine the proposed method on a certain case, let us focus on the wake of the small bar at the $X_{1} C_{1}$ station. The index of the OMD mode corresponding to the considered coherent structure is denoted by $s h$ (i.e. $n=s h$ ) hereafter. The shape of $\Phi_{s h}^{O M D}$ is shown in figure $12 \mathrm{~b}$ while a spectrum of $d_{s h}$ is presented in figure 14 . The spectrum has a peak at the shedding frequency, however it is also spread among an entire frequency band, which is rather undesirable because shedding should be localized in the frequency domain. (16) has been applied in order to recover $\tilde{v}_{s h}$. Enveloped spectra of 
the transverse velocity fluctuation and its residual stochastic part (namely $v-\bar{v}$ and $\left.v-\bar{v}-\tilde{v}_{s h}\right)$ are compared in figure 14 . The shedding peak has been cancelled out entirely from the residual spectrum. Moreover, no artificial suppression of the residual, stochastic fluctuations in the shedding band has been introduced. The second peak, located at 4.6 Hz, hasn't been altered by the procedure which is appreciated since it does not belong to the shedding (see figure 13b for the associated mode). It is an evidence of the method being highly selective although one might have expected that, since the peaks are not well separated in the frequency domain, the method would fail to distinguish between them.

(a)

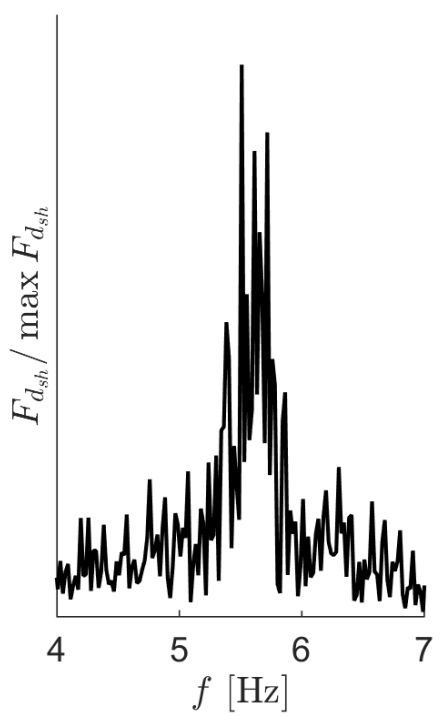

(b)

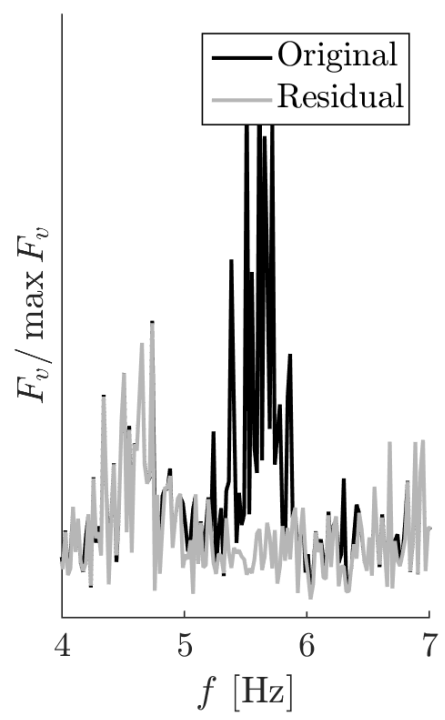

(c)

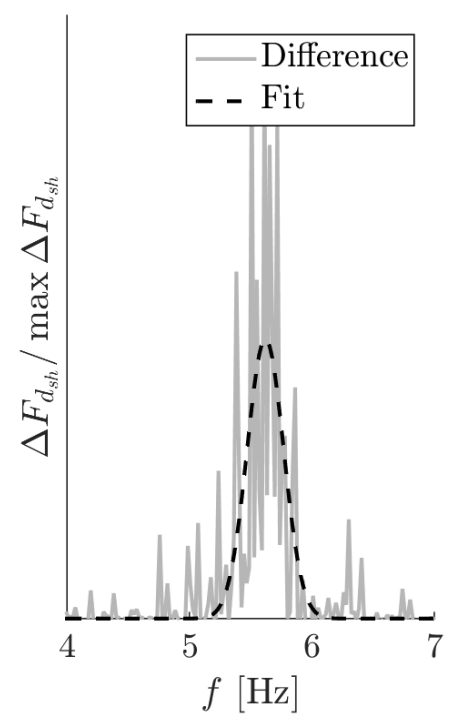

Figure 14: Normalized spectra: a) $d_{s h}$ coefficient spectrum, b) enveloped velocity spectra (original and residual), c) Difference between original and residual enveloped velocity spectrum

Some differences between the original and residual spectra can be seen at all the frequencies, which is a natural consequence of the broad spectrum of $d_{s h}$. This is revealed by subtracting the residual spectrum from the original one. It turns out also, that this procedure clarifies the shedding peak (see figure 14c). In order to limit discrepancies between spectra to a certain frequency interval only a bandpass filter has been applied to $d_{s h}$. The subtracted spectrum signal has been used as the band indicator, i.e. the 
Gaussian curve has been fitted into it and the filter band has been set to the resulting $\mu-3 \sigma<f<\mu+3 \sigma$ interval. The filtered coefficients are referred to as $d_{s h}^{f i l}$ later on. A comparison of spectra produced with $d_{s h}^{f i l}$, given in figure 15 , reveals obviously no differences except those in the prescribed shedding range. The discussed filtering approach is treated as an integral part of the method hereafter, however even with no filter applied the method performs well in terms of distinguishing $\tilde{a}$ and $a^{\prime}$.

(a)

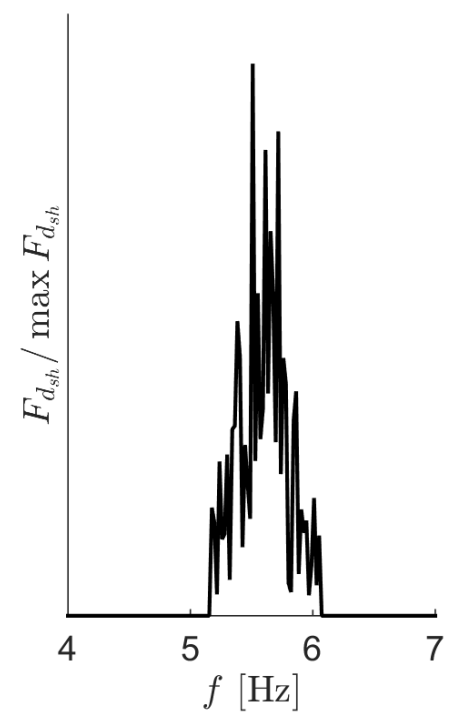

(b)

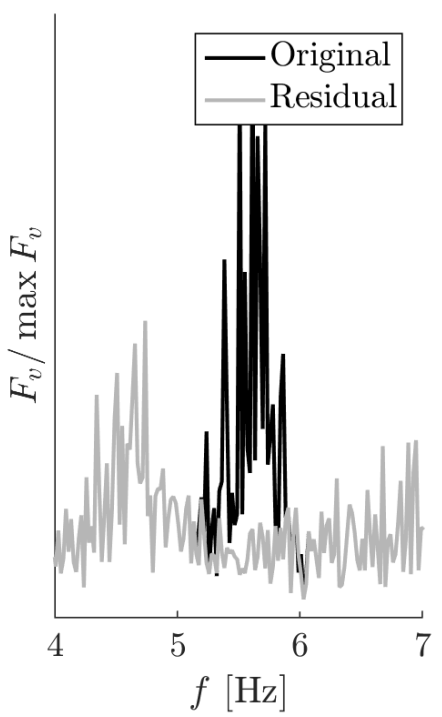

Figure 15: Normalized spectra: a) $d_{s h}^{f i l}$ coefficient spectrum, b) enveloped velocity spectra (original and residual)

It has already been stated that the method does not introduce an artificial suppression at the shedding frequency. This can be explained by the fact that there is motion happening at this frequency having a spatial topology that is different to the one of the corresponding OMD mode and therefore it cannot be removed by subtracting the mode base reconstructed field. The situation can be illustrated with a simple example of a field composed of two sinusoidal spatial modes, e.g. $\mathbf{a}(\mathbf{x}, t)=[\sin (\mathbf{x})+\sin (5 \mathbf{x})] \cdot \sin \left(2 \pi f_{0} \cdot t\right)$. Projecting this field onto either of the modes, say $\Phi(\mathbf{x})=\frac{\sin (\mathbf{x})}{\|\sin (\mathbf{x})\|}$, and then reconstructing 
it one gets:

$$
\tilde{\mathbf{a}}(\mathbf{x}, t)=\Phi^{T} \mathbf{a} \cdot \Phi=\left[1+\frac{\sin (5 \mathbf{x})^{T} \sin (\mathbf{x})}{\|\sin (\mathbf{x})\|^{2}}\right] \cdot \sin (\mathbf{x}) \sin \left(2 \pi f_{0} \cdot t\right)
$$

Obviously the exact value of the second term in the bracket depends on $\mathbf{x}$, however if $\mathbf{x}$ is wide enough, $0<x<2 \pi$ in this case, the second term vanishes. This leads to the conclusion that the residual field $\mathbf{a}-\tilde{\mathbf{a}}$ is washed out of the big scale motion associated with the $f_{0}$ frequency but the small scale one is not affected.

The proposed technique has proven to be successful in extracting the smallest bar shedding at $X_{1} C_{1}$ station, that has been previously recognized as the hardest case within the presented ensemble. Some other results, confirming the method's capability, are presented below. It is worth noting, that the approach provides an instantaneous phase angle value for each wake separately; $\arg \left(d_{s h}^{f i l}\right)$ can be used for recovering this information. Additionally, the method can be employed in the bin averaging approach as the phase indicator in addition.

\subsection{Sensitivity to prescribed parameters}

There are two parameters, which can be set arbitrarily, within the described method: the subspace rank and the filter band. Since there are no strict indications for how to chose either of them, the desired situation is that their influence on the results is weak. The impact of both parameters is briefly presented in this section.

The subspace rank has been arbitrarily limited to $20 \leq r \leq 30$ in the previous section. Let us therefore investigate results for $r \in R=\{20,24,28,32\}$. The first comparison involves mode shapes. Again, the example of the small bar at the $X_{1} C_{1}$ station is employed. Figure 16 presents modes corresponding to different rank values. Except for 16b and $16 \mathrm{c}$, the modes looks different due to different phase position so the comparison is 
(a)

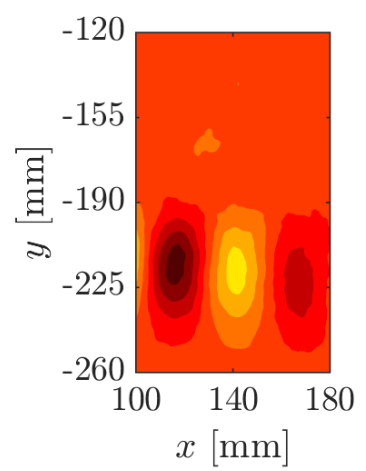

(b)

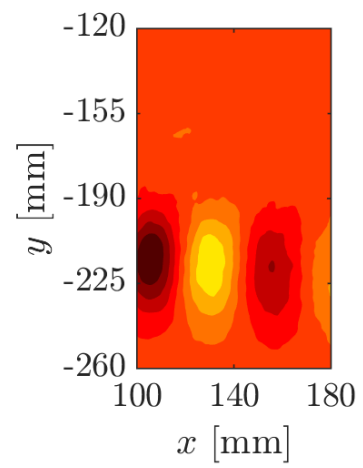

(c)

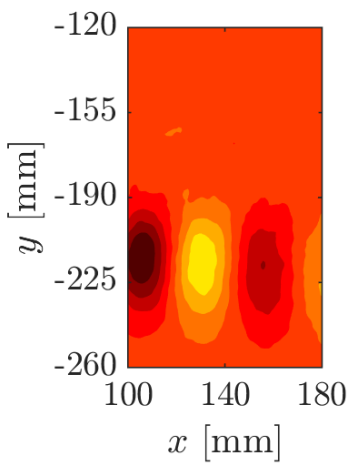

(d)

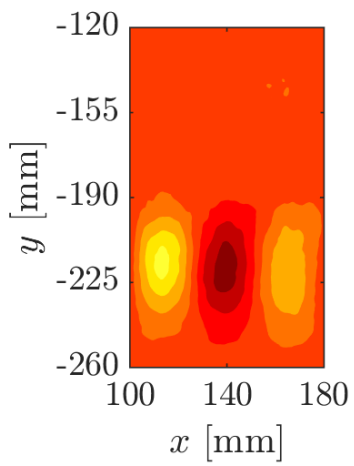

Figure 16: OMD modes corresponding to the small bar shedding evaluated for: a) $r=20$, b) $r=24$, c) $r=28$, d) $r=32$

(a)

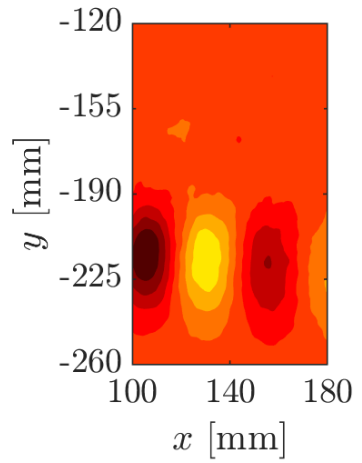

(b)

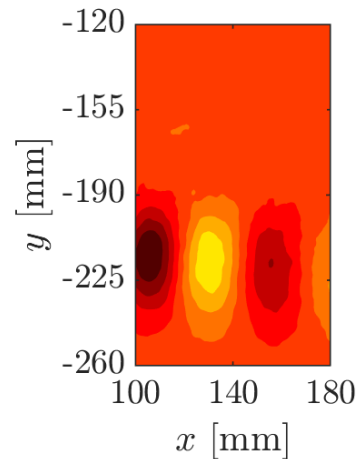

(c)

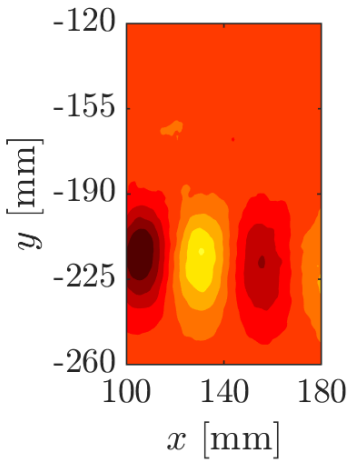

(d)

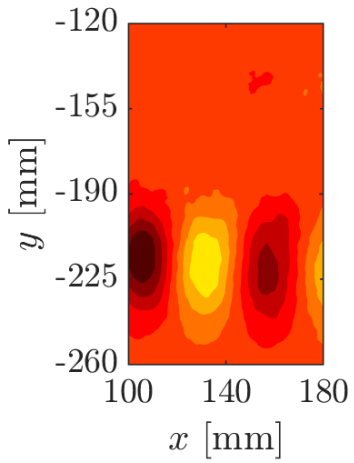

Figure 17: Snapshots reconstructed with OMD modes corresponding to the small bar shedding evaluated for: a) $r=20$, b) $r=24$, c) $r=28$, d) $r=32$

hard to conduct. In order to overcome this situation the reconstructed snapshots corresponding to the same instant of time are attached as well (see figure 17). The similarity is strong, especially in the first three cases. In order to quantify the divergence between the reconstructed snapshots the following parameter has been established:

$$
\begin{gathered}
\hat{\mathbf{v}}_{r . r m s}=\left[\hat{v}_{r . r m s}^{1}, \ldots, \hat{v}_{r . r m s}^{p}\right]^{T} \\
\hat{v}_{r . r m s}^{k}=\sqrt{\frac{1}{N \cdot|R|} \sum_{n=1}^{N} \sum_{r \in R}\left(\hat{v}_{n, r}^{k}-\frac{1}{|R|} \sum_{r \in R} \hat{v}_{n, r}^{k}\right)^{2}}
\end{gathered}
$$

in which $\hat{\mathbf{v}}_{n}^{r}$ stands for 1D representation of $n$-th snapshot reconstructed with the rank $r$ OMD approach and $\hat{v}_{n, r}^{k}$ for its $k$-th element. 
The associated contour map is showed in figure 18. One can observe that $\hat{\mathbf{v}}_{r . r m s}$, which can be understood as a standard deviation of fluctuations of fields reconstructed with different $r$ values around the $r$ averaged field, is one order of magnitude lower compared to the rms value of the transverse velocity field in the wake area (averaged across all the $r$ values).

(a)

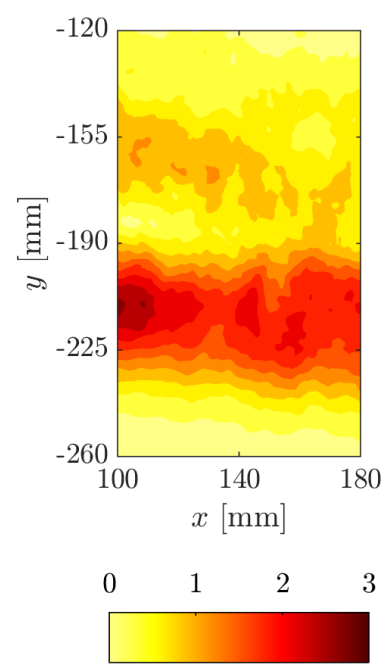

(b)

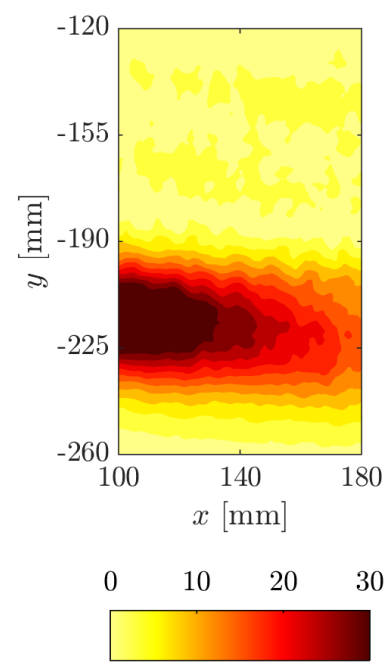

Figure 18: a) the value of $\hat{\mathbf{v}}_{r . r m s}$, b) transverse velocity rms averaged for all considered $r$ values

The discrepancies between phase angle evolution, defined as (19), can be seen in figure 19a. The rms of the angle differences is below $3^{\circ}$ for each $r$ value, almost an order of magnitude less then an usual bin width used in the bin averaging technique (e.g. $22.5^{\circ}$ in the case of Cantwell and Coles [2]), which is important in the context of using the proposed method as a phase indicator. Figure 20 presents the enveloped transverse velocity spectra associated with different ranks, that turn out to be very similar. The coefficient of variance, showed in the same figure, is of the order of $4 \%$ within the $5.2<f<5.8 \mathrm{~Hz}$ range (the shedding band). 


$$
\begin{gathered}
\Delta \phi_{r}(t)=\phi_{r}(t)-\phi^{f i t}(t) \\
\phi^{f i t}(t)=\min _{\phi} \sum_{r}\left[\phi_{r}(t)-\phi\right]^{2}
\end{gathered}
$$

(a)

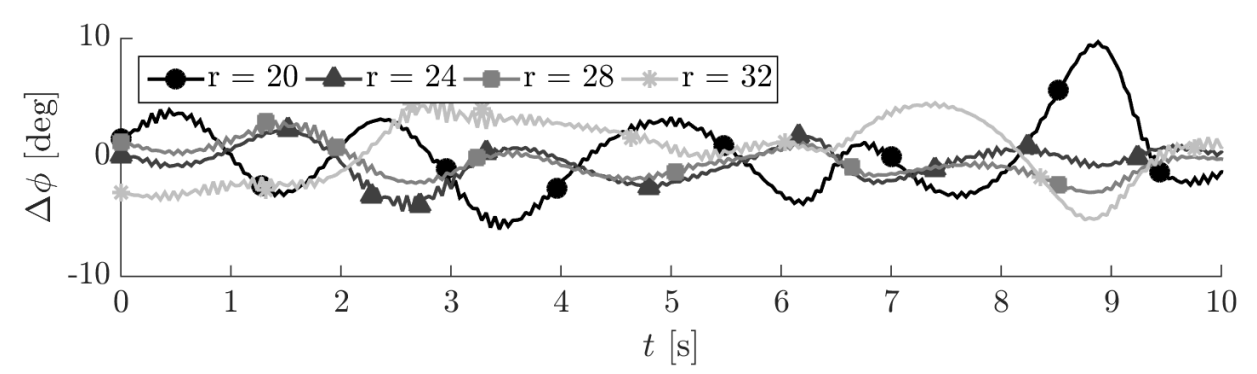

(b)

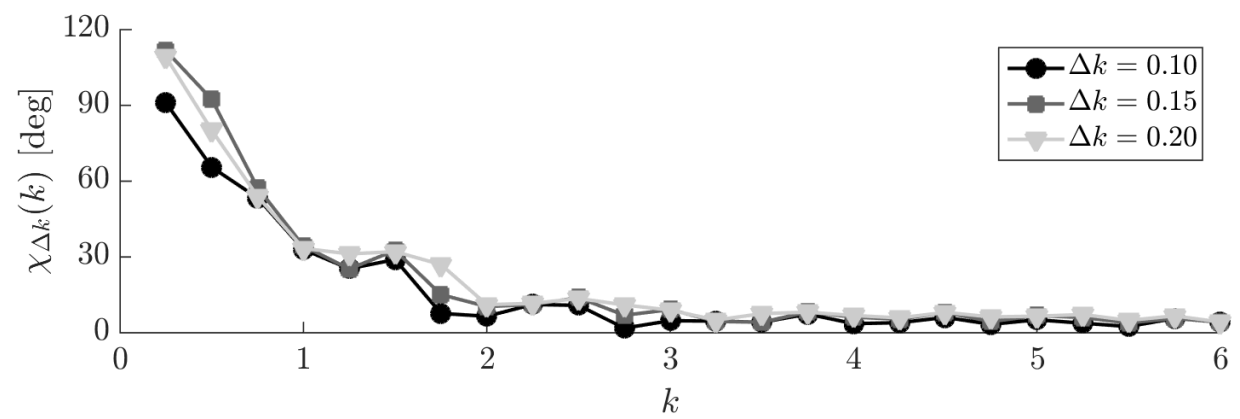

Figure 19: a) Discrepancies in the small bar shedding phase angle evaluated for different $r$ values, b) $\chi_{\Delta k}$ evolution for different $\Delta k$ values

(a)

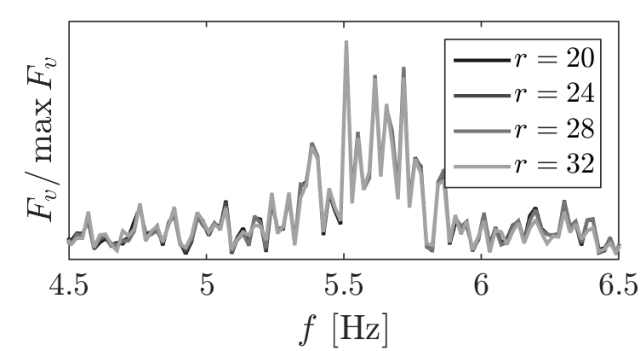

(b)

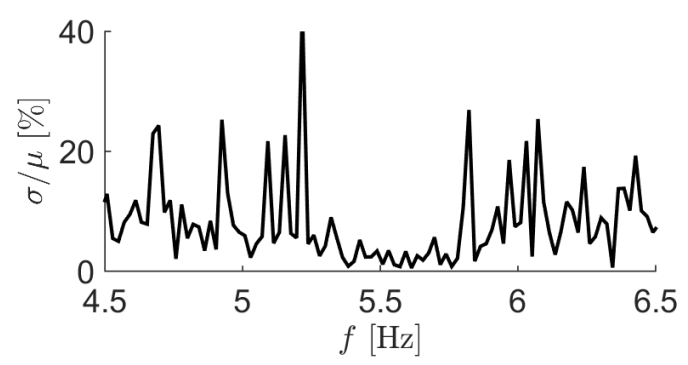

Figure 20: a) transverse velocity spectra of the reconstructed field evaluated for different $r$ values, b) normalized standard deviation of spectra

The second prescribed parameter is the filter band width. The assumption has been made that the Gaussian fit into the difference between the spectra (see figure 14) is a base for the band definition, i.e. it has been set to $\mu \pm k \sigma$ where $k$ is chosen arbitrarily $(k=3$ in the case of our analysis). Employing a standard deviation as a cutoff measure is well 
established in scientific research, nevertheless it is worth checking how the assumed band width affects the results.

It has been observed that the phase angle signal is significantly altered by changing the $k$ value. In order to quantify this effect the following parameter is introduced (the phase signal is treated here as a function of $k$ and denoted by $\left.\phi^{k}\right)$ :

$$
\chi_{\Delta k}(k)=\operatorname{std}\left(\phi^{k+\Delta k}-\phi^{k-\Delta k}\right)
$$

In the ideal case, $\chi_{\Delta k}$ would converge to 0 at a sufficient $k$ value which would mean that $\phi$ doesn't depend on $k$ any longer. This point would define the proper band width. The actual $\chi_{\Delta k}$ evolution, calculated for three different $\Delta k$ values, is presented in figure 19b. The quantity drops significantly with increasing band width, reaching a plateau for $k>2.5$ which is established at a level of $\simeq 5^{\circ}$ in the presented cases. The plateau starting point forms the suitable indication for the band width selection. The value of $k$ assumed in our calculations seems to be a good choice from this perspective. On the other hand, $\chi_{\Delta k}$ doesn't reach 0 as desired. This is a direct consequence of $d_{s h}$ having a broad spectrum because the $\phi^{k+\Delta k}-\phi^{k-\Delta k}$ signal depends on the spectrum part banded by $\mu \pm(k-\Delta k) \sigma<f<\mu \pm(k+\Delta k) \sigma$, which is nonzero regardless of $k$ and $\Delta k$ values. A certain plateau level is, therefore, unavoidable, however this is not a major issue since the level is low compared with typical bin widths in the bin averaging technique

The above analysis indicates the robustness of the method, at least with respect to the parameter $r$. The filter band may raise some doubts but the $\pm 3 \sigma$ cutoff seems to be a reasonable choice nevertheless. 


\section{Application examples}

Some interesting results derived with the proposed method are presented here. Let us start with velocity spectra. These are given for both grid I and grid II at four different positions (see figure 21). Shedding peaks have been successfully subtracted in each of the presented situations and the recognizable residual peak is present only in the case of grid II at the $X_{1} C_{2}$ station. Common features of the presented spectra are little side peaks (e.g. at the $X_{1} C_{2}$ station for grid I) remaining after the phase dependent part subtraction however these can be effectively suppressed if one increased the filtering band. Apart from this the spectra appear to be efficiently decoupled. The shedding peaks are quite close to each other in the cases of grid II at stations $X_{0} C_{2}$ and $X_{1} C_{2}(1.8 \mathrm{~Hz}, 2.2 \mathrm{~Hz}$, different frequencies correspond to the bars of the same size as in Yen and Liu [19]). Despite this both peaks have been extracted separately. This can be seen as another benchmark for the proposed method.

The extracted coherent modes can be used to estimate shedding intensity for different bars, i.e. the share of each mode in the total fluctuations energy can be given. This is presented in table 6 . In order to take into account the fact that the reconstructed coherent part $\tilde{v}$ and the residual velocity field $v-\bar{v}-\tilde{v}$ are not orthogonal, the energy content is calculated based on both separately, however the differences are negligible. These results support the comment about wakes separation in the energy domain made while discussing the POD approach, since the energy of the small bar shedding now appears to be smaller by an order of magnitude. It also highlights the OMD capability of capturing a multiscale flow's dynamics.

Three cases of probability density functions (PDF) of the transverse velocity fluctuations are presented in figure 22, all of them derived with the results for grid I at $X_{0} C_{1}$ 
(a)
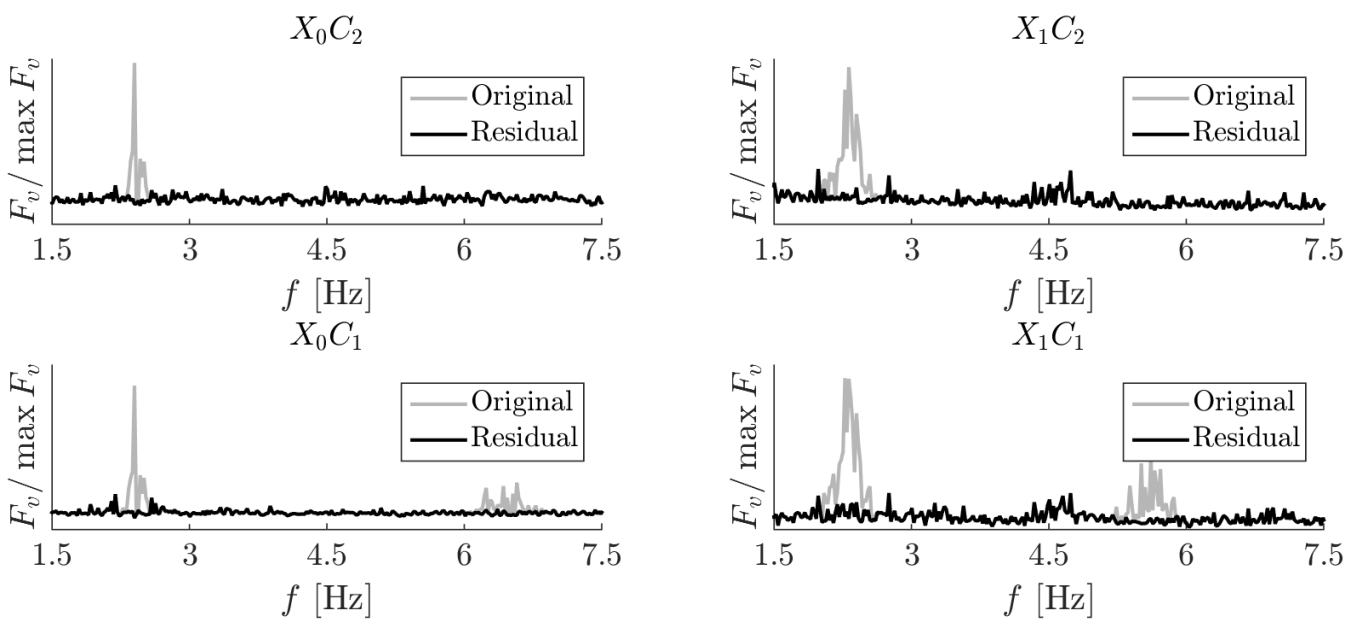

(b)
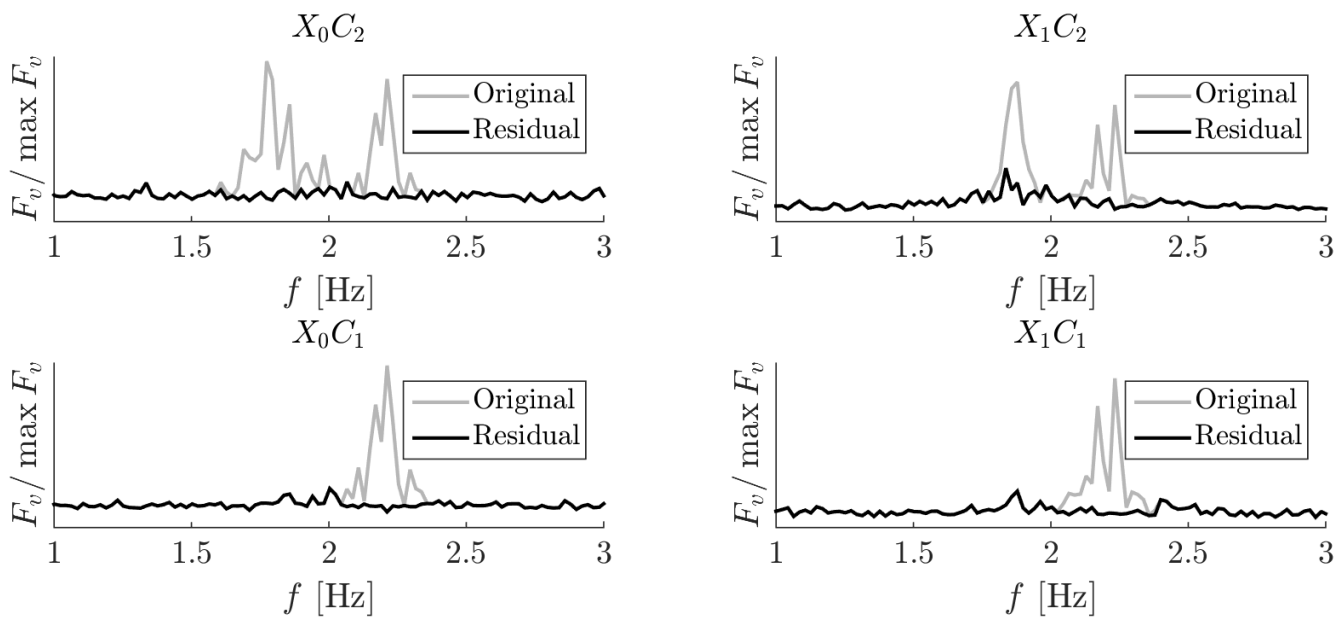

Figure 21: Enveloped transverse velocity spectra of the original and stochastic (residual) data evaluated for: a) grid I, b) grid II

station. The plot correspond to the following locations: outside the wake region, at the centerline of the wake of the small bar and at the centerline of the wake of the medium bar. In the cases of points within wakes the M-shaped PDFs appear for the original data, which is generally expected (the PDF of a sinusoidal signal is M-shaped). Importantly, the residual PDFs (the stochastic part of the fluctuations) recall the sub-Gaussian shaped PDF of turbulent flow (see Wilczek et al. [20] for instance) much more in both cases. On the other hand, the original PDF was only slightly altered by the procedure in the case of the point outside the wake. This is another proof of the capability of the proposed method. 
Table 6: The relative energy (based on transverse velocity) of sheddings of grid I bars (the medium and the small bar indexes are 1 and 2 respectively) calculated at different downstream stations

\begin{tabular}{c|cc|cc} 
& $E\left(\tilde{v}_{1}\right)$ & $1-E\left(v-\bar{v}-\tilde{v}_{1}\right)$ & $E\left(\tilde{v}_{2}\right)$ & $1-E\left(v-\bar{v}-\tilde{v}_{2}\right)$ \\
\hline$X_{0} C_{1}$ & $21.6 \%$ & $22.8 \%$ & $5.2 \%$ & $5.2 \%$ \\
$X_{1} C_{1}$ & $35.1 \%$ & $37.0 \%$ & $3.2 \%$ & $3.2 \%$
\end{tabular}

(a)

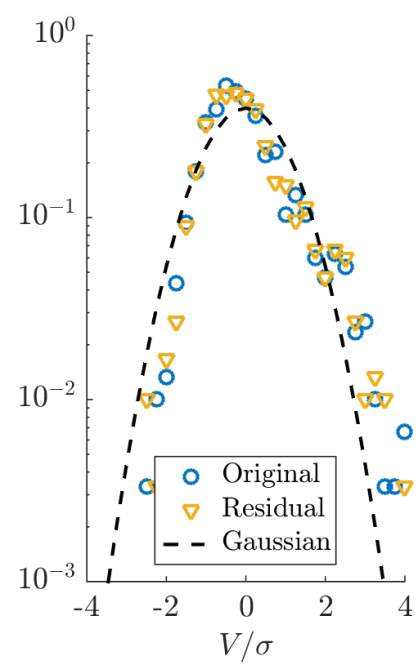

(b)

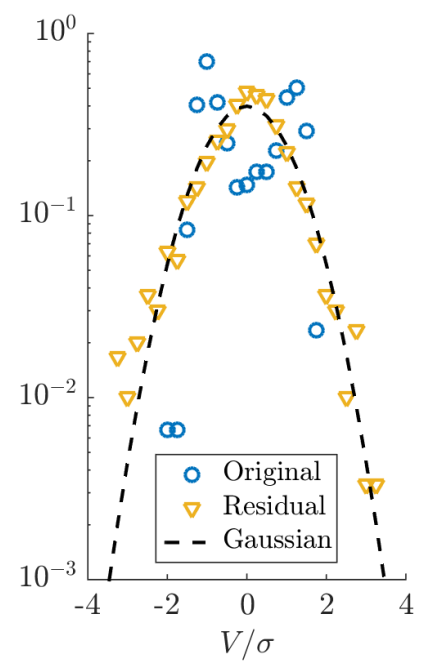

(c)

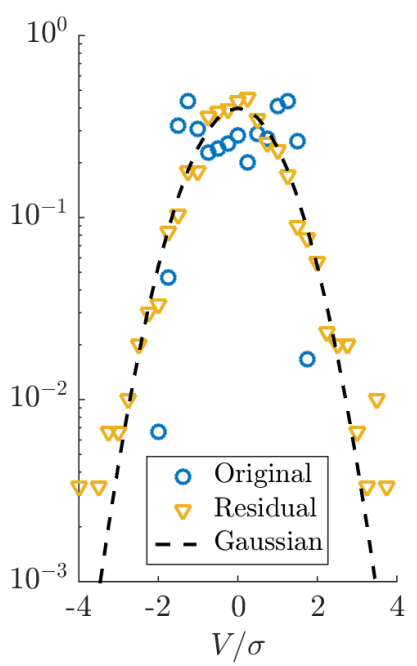

Figure 22: PDFs of the transverse velocity fluctuations evaluated for the original, reconstructed (coherent) and residual (stochastic) data at: a) point outside a wake, b) point inside the wake of the small bar, c) point inside the wake of the medium bar

An example of a more sophisticated analysis is given here. The transverse integral length scale has been studied as a function of the phase angle of the vortex shedding of the small bar. The following formulation has been utilized:

$$
\begin{gathered}
L_{v}\left(x_{0}, y_{0}, \phi\right)=\int_{-\infty}^{+\infty} \tilde{R}_{21}\left(x_{0}, y_{0}, \xi, \phi\right) \mathrm{d} \xi \\
\tilde{R}_{21}\left(x_{0}, y_{0}, \xi, \phi\right)=\frac{\frac{u^{\prime}\left(x_{0}, y_{0}, t(\phi+2 \pi k)\right) \cdot u^{\prime}\left(x_{0}, y_{0}+\xi, t(\phi+2 \pi k)\right)}{\overline{u^{\prime}\left(x_{0}, y_{0}, t(\phi+2 \pi k)\right)^{2}}}}{}
\end{gathered}
$$

where $L_{v}$ is the phase specific transverse integral length scale, $\tilde{R}_{21}$ is similar to the component of a correlation tensor and $u^{\prime}$ stands for stochastic longitudinal velocity fluctuation. The difference between $\tilde{R}_{21}$ and a proper correlation tensor element $R_{21}$ is that the averaging procedure present in the tensor definition is applied over instants of time associated 
with the same phase position of the corresponding coherent part only. Since the assumption of $u^{\prime}$ being isotropic and homogeneous doesn't hold in our case, $\tilde{R}_{21}$ is a function of the spatial position $\left(x_{0}, y_{0}\right)$ and there is also no reason for $\tilde{R}_{21}$ to be symmetric with respect to the points' separation distance (denoted by $\xi$ ). This implies a necessity of integrating $\tilde{R}_{21}$ in $[-\infty,+\infty]$ bounds rather than the commonly employed $[0,+\infty]$.

Since the direct application of (21) requires a huge number of snapshots, the bin averaging approach has been employed to evaluate $\tilde{R}_{21}$, i.e. averaging over distinct phase positions has been replaced with averaging over the corresponding phase bins (the bin size was set to $0.2 \mathrm{rad}$ in our case). The proposed OMD approach has been used to extract $u^{\prime}$ and to provide the phase reference. The presented results correspond to the stations $X_{0} C_{1}$ and $X_{1} C_{2}$ so wakes of both bars appear there but $\tilde{R}_{21}$ has been conditioned on the phase angle of the small bar shedding only. Obviously, integration with infinite bounds is numerically impossible, so it has been replaced with integration up to the first zero crossing.

(a)

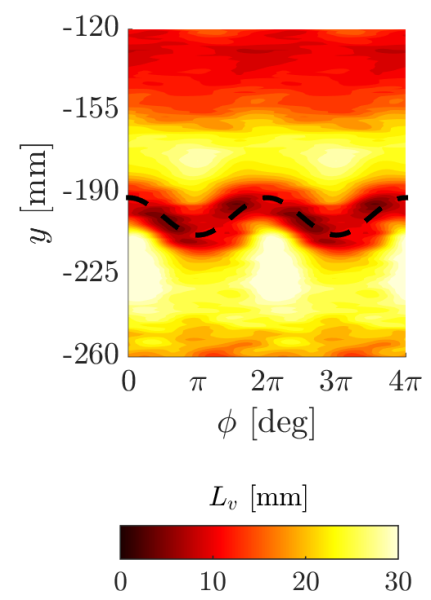

(b)

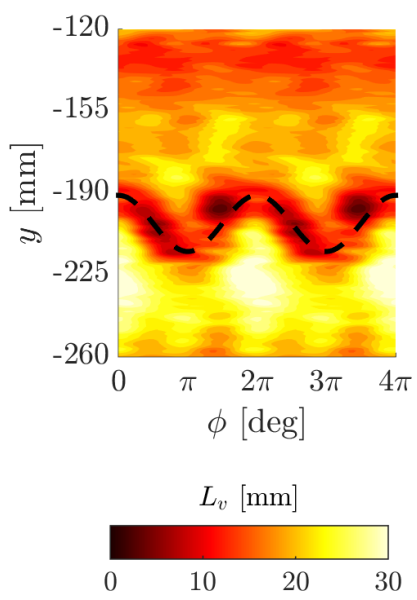

(c)

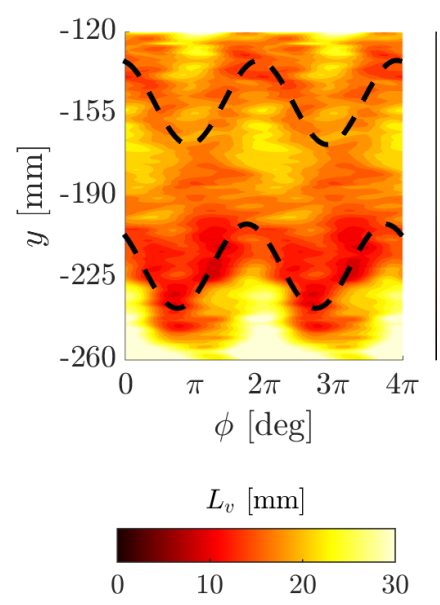

Figure 23: Transverse integral length scale as a function of the phase angle of the shedding of the small bar evaluated at (positions normalized with the small bar thickness): a) $\frac{X}{\delta}=3.1$, b) $\frac{X}{\delta}=5.2$, c) $\frac{X}{\delta}=10.4$

Results at three separate downstream locations are presented in figure 23. The first 
observation is that the properties of the stochastic part (the transverse integral length scale in this case) depend on the coherent part of the fluctuation. Otherwise there would be no evolution in the presented plots along the phase direction. Figure 23a corresponds to the location where the wakes have not yet intersected (see figure 3). The sinusoidal valley of the small bar wake of approximately constant width is clearly distinguishable. $\frac{L_{v}}{\delta}$ is of order of 0.63 (normalized with the small bar thickness) there which is approximately 3-4 times smaller than the wake width. On the other hand there is almost no dependance on the phase angle within the second wake. It is expected since the averaging was conditioned on the smaller wake phase. The integral scale measured between wakes oscillates reaching $25 \mathrm{~mm}$ for phase angles $\{0,2 \pi, \ldots\}$ up to $30 \mathrm{~mm}$ for $\{\pi, 3 \pi, \ldots\}$. These are almost precisely the values of separation between wakes corresponding to those phase positions. This is again an expected result since there is the region of potential flow (i.e. $v^{\prime} \approx 0$ ) bounded by wakes at the early downstream positions and the integral scale in the laminar flow is infinite or, as in this case, is of order of the size of the domain.

Figures $23 \mathrm{~b}$ and $23 \mathrm{c}$ correspond to the locations slightly upstream and downstream of the wakes' intersection point. The interesting result, which is better established in the last plot, is that some structure seems to appear in the bigger wake (tagged with a dashed line in the figure). This means that some phase locking between the wakes takes place. A satisfactory explanation for this phenomenon is not yet available. Perhaps it can be linked to the shedding frequency shift observed for the small bar or maybe it is a form of manifestation of the space scale unfolding (SSU) mechanism postulated by Hurst and Vassilicos [9]. Further research is planned in order to properly document and explain this effect. Despite the mentioned lack of knowledge, it is clear that the proposed method is a useful tool in the study of multiscale flow. 


\section{Conclusions}

It has been shown that the triple decomposition in the form postulated for a multiscale flow, namely (4), can be effectively applied to DNS/experimental data by means of the proposed OMD based approach. The coherent structures corresponding to different scales, $\tilde{a}_{n}$, can be directly linked to particular OMD modes. The efficiency of the decomposition is reflected in the spectra of the turbulent fluctuations, whose peaks due to coherence seem to be almost perfectly leveled in the presented cases while the stochastic fluctuations stay almost unaffected. The method is capable of providing a phase reference signal for each structure separately, which is a non-trivial task in the case of a multiscale flow. This is done purely based on the velocity field without relying on any additional measurements which is beneficial in many ways (it saves time and effort, limits the jitter impact on the results, avoids phase lags, takes into account the fact that coherent events are represented by frequency modulation in certain bands rather than by peaks at distinctive frequencies, etc.). The method can be thus used as a phase indication technique in the bin averaging or in various types of phase conditioned studies. It is worth noting that the method is not limited to a velocity field, as it can be equally applied to other fields (e.g. vorticity, scalar concentration), either separately or simultaneously.

The robustness of the method has also been investigated. The arbitrary value of the subspace rank has been found to affect the results only slightly within the scanned range. The filtering procedure remains to be a minor issue since the resulting phase angle is altered when the band is changed, although the effect is small for a suitably chosen band width. Nevertheless, the proposed method performs the decomposition according to (4) in a multiscale flow in a relatively easy and reliable way which is of major significance. 


\section{Acknowledgements}

The authors acknowledge support form the EU through the FP7 Marie Curie MULTISOLVE project (grant agreement No. 317269). We are grateful to Dr Andrew Wynn for his helpful comments on an earlier version of the manuscript. The MATLAB implementation of the OMD algorithm was downloaded from URL http://control.ee.ethz.ch/ index.cgi?page=publications\&action=details\&id=4094.

\section{References}

[1] A. K. M. F. Hussain and W. C. Reynolds. The mechanics of an organized wave in turbulent shear flow. Journal of Fluid Mechanics, 41(02):241-258, 1970.

[2] B. Cantwell and D. Coles. An experimental study of entrainment and transport in the turbulent near wake of a circular cylinder. Journal of fluid mechanics, 136:321-374, 1983.

[3] R. Sonnenberger, K. Graichen, and P. Erk. Fourier averaging: a phase-averaging method for periodic flow. Experiments in fluids, 28(3):217-224, 2000.

[4] G. Brereton and A. Kodal. A frequency-domain filtering technique for triple decomposition of unsteady turbulent flow. Journal of fluids engineering, 114(1):45-51, 1992.

[5] R. Perrin, M. Braza, E. Cid, S. Cazin, F. Moradei, A. Barthet, A. Sevrain, and Y. Hoarau. Near-wake turbulence properties in the high Reynolds number incompressible flow around a circular cylinder measured by two-and three-component PIV. Flow, turbulence and combustion, 77(1-4):185-204, 2006. 
[6] R. J. Adrian. Conditional eddies in isotropic turbulence. Physics of Fluids (19581988), 22(11):2065-2070, 1979.

[7] A. Grossmann and J. Morlet. Decomposition of Hardy functions into square integrable wavelets of constant shape. SIAM journal on mathematical analysis, 15(4): 723-736, 1984.

[8] N. E. Huang, Z. Shen, S. R. Long, M. C. Wu, H. H. Shih, Q. Zheng, N.-C. Yen, C. C. Tung, and H. H. Liu. The empirical mode decomposition and the Hilbert spectrum for nonlinear and non-stationary time series analysis. Proceedings of the Royal Society of London. Series A: Mathematical, Physical and Engineering Sciences, 454(1971): 903-995, 1998.

[9] D. Hurst and J. Vassilicos. Scalings and decay of fractal-generated turbulence. Physics of Fluids (1994-present), 19(3):035103, 2007.

[10] S. Laizet and J. Vassilicos. Fractal space-scale unfolding mechanism for energyefficient turbulent mixing. Physical Review E, 86(4):046302, 2012.

[11] A. Wynn, D. Pearson, B. Ganapathisubramani, and P. Goulart. Optimal mode decomposition for unsteady flows. Journal of Fluid Mechanics, 733:473-503, 2013.

[12] J. Sung and J. Yoo. Three-dimensional phase averaging of time-resolved PIV measurement data. Measurement Science and Technology, 12(6):655, 2001.

[13] G. Romano, R. Antonia, and T. Zhou. Evaluation of LDA temporal and spatial velocity structure functions in a low Reynolds number turbulent channel flow. Experiments in fluids, 27(4):368-377, 1999. 
[14] A. Okajima. Strouhal numbers of rectangular cylinders. Journal of Fluid Mechanics, 123:379-398, 1982.

[15] G. West and C. Apelt. The effects of tunnel blockage and aspect ratio on the mean flow past a circular cylinder with Reynolds numbers between 104 and 105. Journal of Fluid Mechanics, 114:361-377, 1982.

[16] G. Berkooz, P. Holmes, and J. L. Lumley. The proper orthogonal decomposition in the analysis of turbulent flows. Annual review of fluid mechanics, 25(1):539-575, 1993.

[17] M. D. Graham and I. G. Kevrekidis. Alternative approaches to the Karhunen-Loeve decomposition for model reduction and data analysis. Computers $\&$ chemical engineering, 20(5):495-506, 1996.

[18] P. J. Schmid. Dynamic mode decomposition of numerical and experimental data. Journal of Fluid Mechanics, 656:5-28, 2010.

[19] S. C. Yen and J. H. Liu. Wake flow behind two side-by-side square cylinders. International Journal of Heat and Fluid Flow, 32(1):41-51, 2011.

[20] M. Wilczek, A. Daitche, and R. Friedrich. On the velocity distribution in homogeneous isotropic turbulence: correlations and deviations from gaussianity. Journal of Fluid Mechanics, 676:191-217, 2011. 